\title{
ON SECOND ORDER ELLIPTIC EQUATIONS AND VARIATIONAL INEQUALITIES WITH ANISOTROPIC PRINCIPAL OPERATORS
}

\author{
Vy KHOI LE
}

Abstract. This paper is about boundary value problems of the form

$$
\begin{cases}-\operatorname{div}[\nabla \Phi(\nabla u)]=f(x, u) & \text { in } \Omega, \\ u=0 & \text { on } \partial \Omega,\end{cases}
$$

where $\Phi$ is a convex function of $\xi \in \mathbb{R}^{N}$, rather than a function of the norm $|\xi|$. The problem is formulated appropriately in an anisotropic OrliczSobolev space associated with $\Phi$. We study the existence of solutions and some other properties of the above problem and its corresponding variational inequality in such space.

\section{Introduction}

We are concerned in this paper with the following boundary value problem:

$$
\begin{cases}-\operatorname{div}[\nabla \Phi(\nabla u)]=f(x, u) & \text { in } \Omega, \\ u=0 & \text { on } \partial \Omega,\end{cases}
$$

where $\Omega$ is a bounded region in $\mathbb{R}^{N}(N \geq 1)$ with sufficiently smooth boundary $\partial \Omega$, the function $\Phi: \mathbb{R}^{N} \rightarrow \mathbb{R}$ defines the principal term, and $f: \Omega \times \mathbb{R} \rightarrow \mathbb{R}$ is the lower order term.

2010 Mathematics Subject Classification. 35B45, 35J65, 35J60.

Key words and phrases. Anisotropic Orlicz-Sobolev space, variational inequality, inclusion, multivalued mapping. 
If $\Phi(\xi)=|\xi|^{2} / 2\left(|\cdot|\right.$ is the Euclidean norm in $\left.\mathbb{R}^{N}\right)$ then $\operatorname{div}(\nabla \Phi(\nabla u))=$ $\operatorname{div}(\nabla u)=\Delta u$ is the classical Laplacian. The case $\Phi(\xi)=|\xi|^{p} / p(p>1)$ corresponds to equations with the $p$-Laplacian. In the more general case where $\Phi(\nabla u)=\phi(|\nabla u|)$ where $\phi$ is a convex function from $[0, \infty)$ into itself, we have problems with $\phi$-Laplacian. In the above cases, $\Phi(\xi)$ does not depend on $\xi$ directly but instead on its norm $|\xi|$ and thus the growth of $\Phi$ is the same in all directions (isotropic). In this paper we study equation (1.1) and a more general variational inequality in the anisotropic case, that is, when $\Phi$ has different growths in different directions in $\mathbb{R}^{N}$. This means that $\Phi$ is a function in all components $\xi_{1}, \ldots, \xi_{N}$ of $\xi$, not only in $|\xi|$. A simple example of such function is

$$
\Phi(\xi)=\sum_{j=1}^{N}\left|\xi_{j}\right|^{p_{j}}, \quad \text { for all } \xi=\left(\xi_{1}, \ldots, \xi_{N}\right) \in \mathbb{R}^{N} \text { with } p_{1}, \ldots, p_{n} \in(1, \infty),
$$

or more generally

$$
\Phi(\xi)=\sum_{j=1}^{N} A_{j}\left(\left|\xi_{j}\right|\right), \quad \text { for all } \xi \in \mathbb{R}^{N}
$$

where $A_{1}, \ldots, A_{N}$ are possibly different $N$-functions.

Several interesting problems in Physics and Mechanics have anisotropic natures and have been formulated recently in anisotropic Orlicz spaces or cones, for example in [9] and [18]-[21] (problems in Fluid Mechanics) and in [44], [5] (problems in Elasto-Plasticity). Differential equations with anisotropic operators have been also studied recently in various situations, cf. e.g. [41], [4], [12] and the references therein. After finishing this work, we have learnt of the very recent works [17] and [22] in which similar equations with anisotropic growths were studied in general settings. It was studied in [17] the existence of solutions to the boundary value problem

$$
\begin{cases}-\operatorname{div}(A(x, \nabla u))=f & \text { in } \Omega \\ u=0 & \text { on } \partial \Omega\end{cases}
$$

in generalized Orlicz-Musielak spaces generated by inhomogeneous and anisotropic $N$-functions without $\Delta_{2}$ or $\nabla_{2}$ conditions. In [22], the authors proved the existence of renormalized and weak solutions of the inclusion

$$
\begin{cases}\beta(x, u)-\operatorname{div}(a(x, \nabla u)+F(u)) \ni f & \text { in } \Omega, \\ u=0 & \text { on } \partial \Omega\end{cases}
$$

with $f \in L^{1}(\Omega)$ and $\beta$ being maximal monotone with respect to $u$ in the framework of generalized Orlicz-Musielak spaces. Compared to these interesting works, our approaches, goals, and results here are quite different. More detailed discussions are presented in Remark 3.12. 
In this paper, we propose some steps for a functional analytic study of second order partial differential equations and inclusions with general principal parts having anisotropic growths, that is, different growths in different directions of the gradient and not necessarily given in the form (1.2). We are, in particular, interested in what extend the standard tools in classical cases such as Sobolev spaces or regular Orlicz-Sobolev spaces can be applied in this more general and complicated case and what adaptation and generalization are needed for such extension. We present here basic definitions and properties of anisotropic Orlicz and Orlicz-Sobolev spaces associated with $G$-functions and consider weak formulations of equation (1.1) also in the more general case where $\Phi$ is nonsmooth. As will be seen later, in this case an appropriate formulation of (1.1) is an inclusion or a variational inequality. We study the existence of solutions and properties of the resolvent mapping of (1.1) and its associated variational inequality by extending the classical monotonicity and topological/fixed point methods to our present problems.

The paper is organized as follows. In Section 2, we present the definitions of $G$-functions following [47] and of anisotropic Orlicz and Orlicz-Sobolev spaces associated with $G$-functions, together with some of their basic properties. Section 3 is about weak formulations of (1.1) in anisotropic Orlicz-Sobolev spaces. Abstract existence theorems and properties of resolvent mappings of (1.1) and a more general variational inequality are next established in this general setting. Section 4 is devoted to fixed point formulations of our problems. We show that under certain appropriate growth condition on the lower order term, extending the classical sub-critical growth condition, the problem is equivalent to a fixed point inclusion which has suitable compactness property such that a multivalued Leray-Schauder topological degree for the involved operators is well defined. An illustrative example of the abstract existence theorem in Section 3 when the lower order term has a more specific growth is presented in Section 5 .

\section{Preliminaries}

Assume $\Phi$ is a $G$-function in the sense of [47], that is $\Phi: \mathbb{R}^{N} \rightarrow[0, \infty]$ such that:

(G1) $\Phi(0)=0$,

(G2) $\lim _{|x| \rightarrow \infty} \Phi(x)=\infty$,

(G3) $\Phi$ is convex on $\mathbb{R}^{N}$,

(G4) $\Phi$ is symmetric, i.e. $\Phi(-x)=\Phi(x)$, for all $x \in \mathbb{R}^{N}$,

(G5) The set $\Phi^{-1}=\left\{x \in \mathbb{R}^{N}: \Phi(x)=\infty\right\}$ is separated from 0 ,

(G6) $\Phi$ is lower semicontinuous.

In what follows, we need stronger conditions than (G2) and (G5). In fact, instead of (G2) and (G5), we assume that 
(G2') $\lim _{|x| \rightarrow \infty} \frac{\Phi(x)}{|x|}=\infty$, and

(G5') $\Phi^{-1}(\infty)=\emptyset$, i.e. $\Phi(x) \neq \infty$ for all $x \in \mathbb{R}^{N}$.

In short, we assume that $\Phi$ is a symmetric, convex, lower semicontinuous function from $\mathbb{R}^{N}$ to $[0, \infty)$ that satisfies (G1) and (G2') (cf. Hypothesis 2.1, [9], Definitions 2.1-2.2, [46], and [47]). As a consequence of (G5') and (G6), $\Phi$ is locally Lipschitz and therefore continuous on $\mathbb{R}^{N}$. Let $\Phi^{*}$ be the (Fenchel) conjugate of $\Phi$,

$$
\Phi^{*}(y)=\sup _{x \in \mathbb{R}^{N}}[x \cdot y-\Phi(x)], \quad y \in \mathbb{R}^{N} .
$$

Our assumptions on $\Phi$ imply that $\Phi^{*}$ also satisfies (G1), (G2'), (G3), (G4), (G5'), (G6). It follows from the definition of $\Phi^{*}$ that $x \cdot y \leq \Phi(x)+\Phi^{*}(y)$, for all $x, y \in \mathbb{R}^{N}$ and for each $x \in \mathbb{R}^{N}$, there exists $y \in \mathbb{R}^{N}$ such that

$$
x \cdot y=\Phi(x)+\Phi^{*}(y) .
$$

Furthermore (cf. e.g. [3]),

$$
\Phi^{* *}=\Phi .
$$

We have definitions about some behaviors of $G$-functions.

Definition 2.1. (a) A $G$-function $G$ is said to satisfy a $\Delta_{2}$ condition (at $\infty$ ) if there exist $L, M \geq 0$ such that

$$
\Phi(2 x) \leq L \Phi(x), \quad \text { for all } x \in \mathbb{R}^{N} \text { with }|x| \geq M .
$$

If $M=0$ in (2.4) then we say that $\Phi$ satisfies a $\Delta_{2}$ condition globally.

(b) Given $G$-functions $G_{1}, G_{2}: \mathbb{R}^{N} \rightarrow[0, \infty]$. We say that $G_{1} \prec G_{2}$ if there exist $L, M \geq 0$ such that

$$
G_{1}(x) \leq G_{2}(L x), \quad \text { for all } x \in \mathbb{R}^{N} \text { with }|x| \geq M .
$$

If $G_{1} \prec G_{2}$ and $G_{2} \prec G_{1}$ then we say that $G_{1}$ and $G_{2}$ are equivalent and denote $G_{1} \sim G_{2}$.

We define now the anisotropic Orlicz space $L_{G}(\Omega)$ and first order SobolevOrlicz space $W^{1} L_{G}(\Omega)$. Let $L^{0}(\Omega)$ be the set of all (equivalent classes of) measurable functions from $\Omega$ to $\mathbb{R}$.

Definition 2.2. (a) Let $G: \mathbb{R}^{N} \rightarrow[0, \infty]$ be a $G$-function. The anisotropic Orlicz space associated with $G$ is defined as:

$$
L_{G}(\Omega)=\left\{u \in\left[L^{0}(\Omega)\right]^{N}: \text { exists } \lambda>0 \text { such that } \int_{\Omega} G(\lambda u) d x<\infty\right\} .
$$

(b) Let $G: \mathbb{R}^{N+1} \rightarrow[0, \infty]$ be a $G$-function. The first order anisotropic Orlicz-Sobolev space $W^{1} L_{G}(\Omega)$ associated with $G$ is defined as the set of all weakly differentiable functions $u: \Omega \rightarrow \mathbb{R}$ such that $\left(u, \partial_{1} u, \ldots, \partial_{N} u\right) \in L_{G}(\Omega)$. 
$L_{G}(\Omega)$, equipped with the Luxemburg norm

$$
\|u\|_{L_{G}(\Omega)}=\|u\|_{G}=\inf \left\{\lambda>0: \int_{\Omega} G\left(\frac{u}{\lambda}\right) d x \leq 1\right\} \quad\left(u \in L_{G}(\Omega)\right),
$$

is a Banach space. In Definition 2.2(b), $W^{1} L_{G}$ with the norm

$$
\|u\|_{W^{1} L_{G}(\Omega)}=\|u\|_{1, G}=\left\|\left(u, \partial_{1} u, \ldots, \partial_{N} u\right)\right\|_{G} \quad\left(u \in W^{1} L_{G}\right),
$$

can be identified with a closed subspace of $L_{G}(\Omega)$ and is therefore a Banach space itself. It is clear that $C_{0}^{1}(\Omega) \subset W^{1} L_{G}(\Omega)$. We have next the following definitions.

Definition 2.3. (a) The space $W_{0}^{1} L_{G}(\Omega)$ is the closure of $C_{0}^{1}(\Omega)$ in $W^{1} L_{G}$ with respect to the norm $\|\cdot\|_{1, G}$.

(b) ([47]) Let $G: \mathbb{R}^{N} \rightarrow[0, \infty]$ be a $G$-function. The space $H^{0}(G, \Omega)$ is defined as the completion of the space $C_{0}^{1}(\Omega)$ with respect to the norm

$$
\|\cdot\|_{H^{0}(G, \Omega)}=\|\nabla(\cdot)\|_{G}: u \mapsto\|\nabla u\|_{G}, \quad \text { for all } u \in C_{0}^{1}(\Omega) .
$$

First, we have the following properties of anisotropic Orlicz spaces and norms, whose proofs are straightforward and thus omitted.

Proposition 2.4. Let $G_{1}, G_{2}$ be $G$-functions.

(a) If $G_{1} \prec G_{2}$ then $L_{G_{2}}(\Omega) \subset L_{G_{1}}(\Omega)$ with continuous embedding.

(b) If $G_{1} \sim G_{2}$ then $L_{G_{2}}(\Omega)=L_{G_{1}}(\Omega)$ and the norms $\|\cdot\|_{G_{1}}$ and $\|\cdot\|_{G_{2}}$ are equivalent.

(c) If $G_{1} \prec G_{2}$ and $G_{1}$ satisfies condition (G5') then $G_{2}^{*} \prec G_{1}^{*}$.

Proposition 2.5. Assume $G_{i}: \mathbb{R}^{N_{i}} \rightarrow[0, \infty]$ is a $G$-function $(i=1,2)$. Then $G: \mathbb{R}^{N} \equiv \mathbb{R}^{N_{1}} \times \mathbb{R}^{N_{2}} \rightarrow[0, \infty], G(x)=G\left(x_{1}, x_{2}\right)=G_{1}\left(x_{1}\right)+G_{2}\left(x_{2}\right)$, for all $x=\left(x_{1}, x_{2}\right) \in \mathbb{R}^{N}\left(N=N_{1}+N_{2}, x_{i} \in \mathbb{R}^{N_{i}}, i=1,2\right)$, is a $G$-function on $\mathbb{R}^{N}$ and

(a) $L_{G}(\Omega)=L_{G_{1}}(\Omega) \times L_{G_{2}}(\Omega)$.

(b) For $u=\left(u_{1}, u_{2}\right) \in L_{G}(\Omega)$ with $u_{i} \in L_{G_{i}}(\Omega), i=1,2$, the norms $u \mapsto\|u\|_{G}$ and $u \mapsto\left\|u_{1}\right\|_{G_{1}}+\left\|u_{2}\right\|_{G_{2}}$ are equivalent.

Next, let us consider some relations between the spaces introduced in Definition 2.3. Assume $G: \mathbb{R}^{N} \rightarrow[0, \infty]$ is a $G$-function. Define $G_{1}: \mathbb{R}^{N+1} \rightarrow[0, \infty]$ by

$$
G_{1}\left(x_{0}, x\right)=\left|x_{0}\right|+G(x), \quad \text { for all } x_{0} \in \mathbb{R}, x \in \mathbb{R}^{N} .
$$

Then $G_{1}$ is a $G$-function with

$$
H^{0}(G, \Omega)=W_{0}^{1} L_{G_{1}}(\Omega),
$$

and moreover, the norm $u \mapsto\|u\|_{W^{1} L_{G_{1}}(\Omega)}$ is equivalent to the norm

$$
u \mapsto\|\nabla u\|_{L_{G}(\Omega)},
$$


in this space. In fact, straightforward calculations show that $G_{1}$ is a $G$-function. To verify (2.9), let us assume $u \in H^{0}(G, \Omega)$. Then there exists a sequence $\left\{u_{n}\right\} \subset C_{0}^{1}(\Omega)$ such that $\left\|u_{n}-u\right\|_{H^{0}(G, \Omega)}=\left\|\nabla u_{n}-\nabla u\right\|_{L_{G}(\Omega)} \rightarrow 0$. We know $([47])$ that $H^{0}(G, \Omega) \hookrightarrow W_{0}^{1,1}(\Omega)$ (with continuous embedding) and $\|v\|_{L^{1}(\Omega)} \leq$ $C\|v\|_{H^{0}(G, \Omega)}$, for all $v \in H^{0}(G, \Omega)$ for some constant $C>0$. Hence for some $C_{1}, C_{2}>0$ independent of $u, u_{n}$,

$\left\|u_{n}-u\right\|_{W^{1} L_{G_{1}}(\Omega)} \leq C_{1}\left(\left\|u_{n}-u\right\|_{L^{1}(\Omega)}+\left\|\nabla u_{n}-\nabla u\right\|_{L_{G}(\Omega)}\right) \leq C_{2}\left\|\nabla u_{n}-\nabla u\right\|_{L_{G}(\Omega)}$.

This shows that $u_{n} \rightarrow u$ with respect to the norm $\|\cdot\|_{W^{1} L_{G_{1}}(\Omega)}$, i.e. $u \in$ $W_{0}^{1} L_{G_{1}}(\Omega)$. Conversely, assume $u \in W_{0}^{1} L_{G_{1}}(\Omega)$, i.e. there exists $\left\{u_{n}\right\} \subset C_{0}^{1}(\Omega)$ such that $\left\|u_{n}-u\right\|_{W^{1} L_{G_{1}}(\Omega)} \rightarrow 0$.

Because of the equivalence between $\|\cdot\|_{W^{1} L_{G_{1}}(\Omega)}$ and the norm in (2.10) (see Proposition 2.5), we see that $\left\|u_{n}-u\right\|_{H^{0}(G, \Omega)}=\left\|\nabla u_{n}-\nabla u\right\|_{L_{G}(\Omega)} \rightarrow 0$, i.e. $u \in H^{0}(G, \Omega)$, which completes the proof of $(2.9)$. These arguments also prove the equivalence between the norms $\|\cdot\|_{W^{1} L_{G_{1}}(\Omega)}$ and $\|\nabla(\cdot)\|_{L_{G}(\Omega)}$.

In what follows, we concentrate on $G$-functions $G: \mathbb{R}^{N+1} \rightarrow[0, \infty]$ with

$$
G\left(u_{0}, u\right)=\Phi_{0}\left(u_{0}\right)+\Phi(u), \quad u_{0} \in \mathbb{R}, u \in \mathbb{R}^{N},
$$

where $\Phi_{0}$ is a Young function from $\mathbb{R}$ to $[0, \infty]$ and $\Phi$ a $G$-function from $\mathbb{R}^{N}$ to $[0, \infty]$. As in [47], we denote $\Phi_{+}^{*}(u)=\sup \left\{u \cdot v-\Phi(v): v \in \mathbb{R}_{+}^{N}\right\}$ where $\mathbb{R}_{+}^{N}=\left\{v \in \mathbb{R}^{N}: v_{i} \geq 0\right.$, for all $\left.i \in\{1, \ldots, N\}\right\}$. Let $A$ be a Young function that satisfies Theorem 1 in [47] for $H^{0}(G, \Omega)$, i.e. there are continuous, nondecreasing functions $f_{1}, \ldots, f_{N}$ from $[0, \infty)$ into itself such that

$$
G_{+}^{*}\left(0, f_{1}(s), \ldots, f_{N}(s)\right)\left(=\Phi_{+}^{*}\left(f_{1}(s), \ldots, f_{N}(s)\right)\right) \leq s,
$$

for all $s \in[0, \infty)$, and for some $k>0$,

$$
\frac{1}{k} \int_{0}^{t} \frac{d s}{s\left(\prod_{i=1}^{N} f_{i}(s)\right)^{1 / n}} \leq A^{-1}(t), \quad \text { for all } t \geq 0 .
$$

According to Theorem 1 and the remark in pages 29-30 of [47], the embedding

$$
W_{0}^{1} L_{G}(\Omega) \hookrightarrow L_{A}(\Omega)
$$

is continuous with

$$
\|u\|_{L_{A}(\Omega)} \leq C\||\nabla u|\|_{L_{\Phi}(\Omega)}, \quad \text { for all } u \in W_{0}^{1} L_{G}(\Omega) .
$$

From (2.13) we see that if

$$
\Phi_{0} \prec A,
$$

then the norms $\|\cdot\|_{W^{1} L_{G}}$ and $\|\nabla(\cdot)\|_{L_{\Phi}}$ are equivalent on $W_{0}^{1} L_{G}$. In fact, we have from Theorem 1 in [47],

$$
\|u\|_{L_{\Phi_{0}}} \leq C_{1}\|u\|_{L_{A}} \leq C_{2}\|\nabla u\|_{L_{\Phi}},
$$


for all $u \in H^{0}(\Phi, G) \supset W_{0}^{1} L_{\Phi}$. Therefore, the norms $\|\cdot\|_{L_{\Phi_{0}}}+\|\nabla(\cdot)\|_{L_{\Phi}}$ and $\|\nabla(\cdot)\|_{L_{\Phi}}$ are equivalent on $W_{0}^{1} L_{\Phi}$. From Proposition 2.5 , the norms $\|\cdot\|_{L_{\Phi_{0}}}+$ $\|\nabla(\cdot)\|_{L_{\Phi}}$ and $\|\cdot\|_{W^{1} L_{G}}$ are equivalent on $W^{1} L_{G}$. Hence the norms $\|\cdot\|_{W^{1} L_{G}}$ and $\|\nabla(\cdot)\|_{L_{\Phi}}$ are equivalent. These arguments also imply that if (2.15) holds then $H^{0}(\Phi, \Omega)=W_{0}^{1} L_{G}$.

In the particular case where $\Phi$ is given by (1.2), it was proved in [47] that the function $A$ such that (2.12) and thus (2.13) hold can be chosen as

$$
A^{-1}(t)=\int_{0}^{t} \frac{d s}{\bar{m}(s)} \quad(t \geq 0)
$$

where

$$
\bar{m}(s)=s^{1+1 / N}\left[\prod_{j=1}^{N} A_{j}^{-1}\left(\frac{s}{N}\right)\right]^{-1 / N} .
$$

REMARK 2.6. Instead of using the classical embedding theorem in [47], we can use alternatively a sharp embedding theorem due to Cianchi ([7], see also [6], [8]). If $\Phi$ is as above then $A$ can be constructed as follows. Let $\Phi_{\star}:[0, \infty] \rightarrow$ $[0, \infty]$ be defined by

$$
\Phi_{\star}(s)=\sup \left\{t:\left|\left\{\xi \in \mathbb{R}^{N}: \Phi(\xi) \leq t\right\}\right|<C_{n} s^{n}\right\} \quad \text { for } s \geq 0,
$$

where $C_{n}=\pi^{n / 2} \Gamma(1+n / 2)$ is the measure of the $n$-dimensional unit ball. Assume

$$
\int_{0}^{a}\left(\frac{t}{\Phi_{\star}(t)}\right)^{1 /(N-1)} d t<\infty
$$

for some $a>0$ and define $H:[0, \infty) \rightarrow[0, \infty)$ by

$$
H(r)=\left(\int_{0}^{r}\left(\frac{t}{\Phi_{\star}(t)}\right)^{1 /(N-1)} d t\right)^{(N-1) / N} \text { for } r \geq 0 .
$$

Then for the Young function $A$ given by

$$
A=\Phi_{\star} \circ H^{-1},
$$

where $H^{-1}$ is the left-continuous inverse of $H$, the embedding (2.13) holds (when $\partial \Omega$ is sufficiently smooth). More importantly, the embedding (2.13) is sharp in the class of Young functions with $A$ given by (2.16).

Note that our results in the sequel still hold if $A$ is given by (2.16) instead by (2.11)-(2.12).

Let us conclude this section with a coercivity result for integrals in anisotropic Orlicz spaces that will be used later. This result is obvious for Lebesgue spaces and was proved in [16] for Orlicz spaces. 
Proposition 2.7. Assume $G$ is a $G$-function that satisfies (G2') and (G5'). If $G^{*}$ satisfies a $\Delta_{2}$ condition (at infinity) then $\int_{\Omega} G(u) d x$ is coercive on $L_{G}(\Omega)$ in the following sense:

$$
\lim _{\|u\|_{G} \rightarrow \infty} \frac{1}{\|u\|_{G}} \int_{\Omega} G(u) d x=\infty .
$$

To prove this proposition, we need a simple result on $G$-functions with $\Delta_{2}$ conditions, a one-dimensional version of which (for $N$-functions) was given in $[26]$.

Proposition 2.8. Assume $G$ is a G-function satisfying condition (G5') and $a \Delta_{2}$ condition at $\infty$. Then there exists a $G$-function $H$ satisfying (G5') and a $\Delta_{2}$ condition globally such that $G \sim H$.

Proof. Assume (2.4) holds with $M>0$. By the continuity of $G, K:=$ $\sup \{G(x):|x| \leq M\}+1 \in[1, \infty)$. Let $B=G^{-1}([0, K])$. Since $G$ is a continuous $G$-function, $B$ is a closed, bounded, convex, symmetric (about 0 ) subset of $\mathbb{R}^{N}$. Since $K>0$, the continuity of $G$ implies that $0 \in B^{\circ}$. It also follows from the convexity of $G$ that

$$
G(x)=K \Leftrightarrow x \in \partial B
$$

Next, we prove that

(2.19) For each $x \in B \backslash\{0\}$, there exists a unique $t=t_{x} \geq 1$

such that $t_{x} x \in \partial B$.

In fact, consider the function $G^{x}: \mathbb{R} \rightarrow[0, \infty), G^{x}(t)=G(t x)$. Since $G^{x}(0)=0$ and $G^{x}(t x) \rightarrow \infty$ as $t \rightarrow \infty$, there must exists $t_{0} \in(0, \infty)$ such that $G^{x}\left(t_{0}\right)=K$, i.e. $t_{0} x \in \partial B$ by (2.18). Moreover, from the convexity of $G$ implies that $G^{x}$ is nondecreasing on $[0, \infty)$, i.e. if $0 \leq t_{1}<t_{2}$ then $G^{x}\left(t_{1}\right) \leq G^{x}\left(t_{2}\right)$. Moreover, if $G^{x}\left(t_{2}\right)>0$ then $G^{x}\left(t_{1}\right)<G^{x}\left(t_{2}\right)$.

This property implies that $t_{0} \geq 1$. In fact, if $t_{0}<1$ then $0<K=G^{x}\left(t_{0}\right) \leq$ $G^{x}(1)$ and thus $K=K^{x}\left(t_{0}\right)<G^{x}(1)=G(x)=K$.

To see the uniqueness of $t_{0}$, assume there are $t_{0}, t_{1}$ such that $1 \leq t_{0}<t_{1}$ and $G^{x}\left(t_{0}\right)=G^{x}\left(t_{1}\right)=K$. This is impossible since $G^{x}\left(t_{1}\right)>0$ and thus $K=G^{x}\left(t_{0}\right)<G^{x}\left(t_{1}\right)=K$. This contradiction proves the uniqueness of $t_{0}$ and thus (2.19).

Let us define $G_{1}: B \rightarrow \mathbb{R}$ as follows. $G_{1}(0)=0$ and for $x \in B \backslash\{0\}$, $G_{1}(x)=K / t_{x}$ where $t_{x}$ is the unique number in $[1, \infty)$ such that $t_{x} x \in \partial B$. It is clear that $G_{1}$ is symmetric and $G_{1}(x)=K$ for all $x \in \partial B$. Moreover,

$G_{1}$ is convex on $B$.

In fact, let $x, y \in B, x=t x_{0}, y=s y_{0}$ with $x_{0}, y_{0} \in \partial B$ and $s, t \in[0,1]$. We have by definition, $G_{1}(x)=t K, G_{1}(y)=s K$. Let $z=\lambda x+(1-\lambda) y$ with $\lambda \in(0,1)$. 
We need to prove that

$$
G_{1}(z) \leq \lambda G_{1}(x)+(1-\lambda) G_{1}(y) .
$$

Without loss of generality, we can assume that either $t>0$ or $s>0$. Consider $\sigma=[\lambda t+(1-\lambda) s]^{-1}$. We have $\sigma \geq 1$ and $\sigma z=\sigma \lambda t x_{0}+\sigma(1-\lambda) s y_{0}$ with $\sigma \lambda t+\sigma(1-\lambda) s=1$ and $\sigma \lambda t, \sigma(1-\lambda) s \geq 0$. Since $B$ is convex, we have $\sigma z \in B$, i.e. $G_{1}(\sigma z) \leq K$. Note that $G_{1}(\sigma z)=\sigma G_{1}(z)$ and thus

$$
G_{1}(z) \leq \frac{K}{\sigma}=\lambda t K+(1-\lambda) s K=\lambda G_{1}(x)+(1-\lambda) G_{1}(y),
$$

proving (2.21) and therefore (2.20). It follows that

$$
\operatorname{epi}\left(G_{1}\right)=\left\{X=\left(x, x^{*}\right) \in B \times \mathbb{R}: G_{1}(x) \leq x^{*}\right\} \text { is convex. }
$$

Let us define $H: \mathbb{R}^{N} \rightarrow[0, \infty)$ by

$$
H(x)= \begin{cases}G_{1}(x) & \text { if } x \in B, \\ G(x) & \text { if } x \notin B .\end{cases}
$$

Straightforward arguments show that $H$ is continuous in $\mathbb{R}^{N}$. Let us prove that $H$ is convex in $\mathbb{R}^{N}$, i.e. to prove that epi $H=\left\{\left(x, x^{*}\right) \in \mathbb{R}^{N+1}: x \in \mathbb{R}^{N}\right.$ and $x \geq$ $H(x)\}$ is a convex set. First, note that

$$
\text { epi } H \subset \text { epi } G \text {. }
$$

In fact, let $\left(x, x^{*}\right) \in$ epi $H$. If $x \notin B$ then $G(x)=H(x)$ and $\left(x, x^{*}\right) \in$ epi $G$. If $x \in B$ with $x=t x_{0}, x_{0} \in \partial B$ and $t \in[0,1]$, we have $\left(x, G_{1}(x)\right)=(x, t K)=$ $t\left(x_{0}, G\left(x_{0}\right)\right)$. Since epi $G$ is convex and contains $(0,0)$, we have $\left(x, G_{1}(x)\right) \in$ epi $G$, i.e. $G_{1}(x) \geq G(x)$. It follows that $x^{*} \geq H(x)=G_{1}(x) \geq G(x)$ and thus $\left(x, x^{*}\right) \in \operatorname{epi} G$, proving $(2.24)$. Let $X=\left(x, x^{*}\right), Y=\left(y, y^{*}\right) \in$ epi $H$. We prove that

$$
[X, Y]:=\{\lambda X+(1-\lambda) Y: \lambda \in[0,1]\} \subset \text { epi } H .
$$

We first note the following equivalences, whose proofs are straightforward and thus omitted. For $Z=\left(z, z^{*}\right) \in \mathbb{R}^{N+1}\left(z \in \mathbb{R}^{N}\right.$ and $\left.z^{*} \in \mathbb{R}\right)$, we have

$$
\left[Z \in \text { epi } H \text { and } z^{*} \geq K\right] \Leftrightarrow\left[Z \in \operatorname{epi} G \text { and } z^{*} \geq K\right],
$$

and

$$
\left[Z \in \text { epi } H \text { and } z^{*} \leq K\right] \Leftrightarrow\left[Z \in \operatorname{epi} G_{1} \text { and } z^{*} \leq K\right] .
$$

To prove (2.25), we consider 3 cases.

(i) Both $x^{*}, y^{*} \geq K$. It follows from (2.26) that $X, Y \in \operatorname{epi} G$. Since epi $G$ is convex, $[X, Y] \subset$ epi $G$. For any $Z=\left(z, z^{*}\right) \in[X, Y]$, since $z^{*}$ is a convex combination of $x^{*}$ and $y^{*}$, we have $z^{*} \geq K$. Thus from (2.26), $Z=\left(z, z^{*}\right) \in$ epi $H$ and (2.25) is proved in this case. 
(ii) Both $x^{*}, y^{*} \leq K$. For this case, the equivalence in (2.27) implies that $X, Y \in \operatorname{epi} G_{1}$. Using again the convexity of epi $G_{1}$ (cf. $\left.(2.22)\right)$, we have $[X, Y] \subset$ epi $G_{1}$, and thus for any $Z=\left(z, z^{*}\right) \in[X, Y]$, we have $z^{*} \leq K$. Since $Z \in \operatorname{epi} G_{1}$, (2.27) implies that $Z \in$ epi $H$.

(iii) $\min \left\{x^{*}, y^{*}\right\} \leq K<\max \left\{x^{*}, y^{*}\right\}$. Assume for example $x^{*} \leq K<y^{*}$. Then there exists a unique $Z=\left(z, z^{*}\right) \in[X, Y]$ such that $z^{*}=K$. It is clear that

$$
[X, Y]=[X, Z] \cup[Z, Y] .
$$

From (2.24), we have $[X, Y] \subset$ epi $G$ and thus $Z \in$ epi $G$. As a consequence, $K=z^{*} \geq G(z)$ and thus $z \in B$. It follows from the definition of $H$ and $G_{1}$ that $H(z)=G_{1}(z)=t K$ where $t \in[0,1]$ is such that $z=t z_{0}, z_{0} \in \partial B$. We thus have $H(z)=t K \leq K=z^{*}$ and thus $Z=\left(z, z^{*}\right)=(z, K) \in$ epi $H$. Since $X, Z \in$ epi $H$ with $x^{*}, z^{*} \leq K$, we have

$$
[X, Z] \subset \text { epi } H
$$

from the proof in case (ii) with $Z$ instead of $Y$. Similarly, since $Z, Y \in$ epi $H$ with $z^{*}, y^{*} \geq K$, we have

$$
[Z, Y] \subset \text { epi } H \text {, }
$$

from case (i) above with $Z$ instead of $X$. From (2.28)-(2.30), we have (2.25) also in case (iii) and thus in all cases. We obtain the convexity of $H$ in $\mathbb{R}^{N}$.

Since $B$ is bounded one can choose $\rho \in(0, \infty)$ such that

$$
B \subset B_{\rho}(0)
$$

Because $H(x)=G(x)$ for all $x \in \mathbb{R}^{N},|x| \geq \rho$, we immediately see that $H \sim G$.

Let us prove now that $H$ satisfies a global $\Delta_{2}$ condition. First, note from its definition that $H(x)>0$ for all $x \in \mathbb{R}^{N} \backslash\{0\}$. Furthermore, from the definitions of $B$ and $K$, we have that $\overline{B_{M}(0)} \subset B$. Let $K_{1}=\max \{H(x):|x| \leq 2 \rho\} \in(0, \infty)$ where $\rho$ satisfies $(2.31)$ and $m_{1}=\min \{H(x): M / 2 \leq|x| \leq \rho\} \in(0, \infty)$.

For any $x \in \mathbb{R}^{N}$, we have the following possibilities: $|x| \leq M / 2, M / 2<|x|<$ $\rho$, and $|x| \geq \rho$.

If $|x| \leq M / 2$ then $x, 2 x \in B$ and $H(2 x)=G_{1}(2 x)=t K$ where $2 x=t x_{0}$, $x_{0} \in \partial B$. Since $x=t x_{0} / 2$, we have $H(x)=G_{1}(x)=t K / 2$. Thus $H(2 x)=$ $2 H(x)$ if $|x| \leq M / 2$.

In the case where $M / 2<|x|<\rho$, we have $H(2 x) \leq K_{1}$ and $H(x) \geq m_{1}$ and thus $H(2 x) \leq\left(K_{1} / m_{1}\right) H(x)$.

If $|x| \geq \rho$ then $x, 2 x \notin B$. In particular, $|x| \geq M$ and from (2.4), we have $H(2 x)=G(2 x) \leq L G(x)=L H(x)$. Combining the above cases, we get

$$
H(2 x) \leq \max \left\{2, K_{1} / m_{1}, L\right\} H(x), \quad \text { for all } x \in \mathbb{R}^{N} .
$$


Proof of Proposition 2.7. First, assume that $G^{*}$ satisfies a global $\Delta_{2}$ condition, that is,

$$
G^{*}(2 x) \leq L G^{*}(x), \quad \text { for all } x \in \mathbb{R}^{N},
$$

for some $L>2$. By mathematical induction, we have

$$
G^{*}\left(2^{k} x\right) \leq L^{k} G^{*}(x), \quad \text { for all } x \in \mathbb{R}^{N} \text { and all } k \in \mathbb{N} .
$$

As in the proof of Lemma 3.14, [16], we define the function $f:[1, \infty) \rightarrow[L, \infty)$ by

$$
f(r)=r\left[(1-r) L^{k+1}+\lambda L^{k+2}\right],
$$

if $r=(1-\lambda) 2^{k}+\lambda 2^{k+1}$, with $\lambda \in[0,1], k \in \mathbb{N} \cup\{0\}$, i.e. $f\left(2^{k}\right)=2^{k} L^{k+1}$ and $f(r) / r$ is affine in each interval $\left[2^{k}, 2^{k+1}\right]$ for $k \in \mathbb{N} \cup\{0\}$. From (2.33) and the convexity of $G^{*}$, we see that

$$
G^{*}(r x) \leq f(r) G^{*}(x), \quad \text { for all } x \in \mathbb{R}^{N} \text { and all } r \in[1, \infty) .
$$

Let $r \geq 1$. From (2.35) and Young's inequality, for each $x \in \mathbb{R}^{N}$, there exists $y \in \mathbb{R}^{N}$ such that

$$
\begin{aligned}
G(x)+G^{*}(y) & =\frac{1}{f(r)}\left(\frac{f(r)}{r} x\right) \cdot(r y) \\
& \leq \frac{1}{f(r)}\left[G\left(\frac{f(r)}{r} x\right)+G^{*}(r y)\right] \leq \frac{1}{f(r)} G\left(\frac{f(r)}{r} x\right)+G^{*}(y) .
\end{aligned}
$$

This proves that

$$
G\left(\frac{f(r)}{r} x\right) \geq f(r) G(x), \quad \text { for all } x \in \mathbb{R}^{N} \text { and all } r \geq 1 .
$$

On the other hand, it follows from the definition of $f$ that $r \mapsto f(r) / r$ is strictly increasing function from $[1, \infty)$ onto $[L, \infty)$. Let $g:[L, \infty) \rightarrow[1, \infty)$ be its inverse. We obtain from (2.36) that

$$
G\left(g^{-1}(r) x\right) \geq f(r) G(x), \quad \text { for all } x \in \mathbb{R}^{N} \text { and all } r \geq 1 .
$$

For $s \geq L$, let $s=g^{-1}(r)=f(r) / r$. We have $f(r)=s g(s)$ and the above inequality becomes

$$
G(s x) \geq \operatorname{sg}(s) G(x), \quad \text { for all } x \in \mathbb{R}^{N} \text { and all } s \geq L .
$$

Let $u \in L_{G}(\Omega)$ with $\|u\|_{G}>L+1$. For all $\varepsilon \in(0,1)$, we have $\|u\|_{G}-\varepsilon>1$ and

$$
\int_{\Omega} G\left(\frac{u}{\|u\|_{G}-\varepsilon}\right) d x>1
$$

For almost every $x \in \Omega$, it follows from (2.37) that

$$
G(u(x)) \geq\left(\|u\|_{G}-\varepsilon\right) g\left(\|u\|_{G}-\varepsilon\right) G\left(\frac{u(x)}{\|u\|_{G}-\varepsilon}\right),
$$


and thus, for all $\varepsilon \in(0,1)$,

$$
\begin{aligned}
\int_{\Omega} G(u) d x & \geq\left(\|u\|_{G}-\varepsilon\right) g\left(\|u\|_{G}-\varepsilon\right) \int_{\Omega} G\left(\frac{u}{\|u\|_{G}-\varepsilon}\right) d x \\
& \geq\left(\|u\|_{G}-\varepsilon\right) g\left(\|u\|_{G}-\varepsilon\right) .
\end{aligned}
$$

Hence,

$$
\int_{\Omega} G(u) d x \geq\|u\|_{G} g\left(\|u\|_{G}\right) \quad \text { for all } u \in L_{G}(\Omega) \text { with }\|u\|_{G}>L+1 .
$$

Since $g(s) \rightarrow \infty$ as $s \rightarrow \infty$, we obtain (2.17).

Assume now that $G^{*}$ only satisfies a $\Delta_{2}$ condition at $\infty$. Since $G$ satisfies (G2') and $G=\left(G^{*}\right)^{*}$, we see that $G^{*}$ satisfies condition (G5') (cf. e.g. Theorems 2.35 and $11.8,[45])$ and is thus continuous on $\mathbb{R}^{N}$. From Proposition 2.8, there exists a $G$-function $H: \mathbb{R}^{N} \rightarrow[0, \infty)$ such that $H$ satisfies a global $\Delta_{2}$ condition and $G^{*} \sim H$. Since both $G^{*}$ and $H$ satisfy condition (G5'), it follows from Proposition 2.4 that $G=\left(G^{*}\right)^{*} \sim H^{*}:=G_{1}$. As $H$ satisfies condition (G5'), its conjugate $G_{1}$ satisfies the coercivity condition (G2'). Also, $G_{1}^{*}=H$ satisfies a global $\Delta_{2}$ condition. By the above proof, we have

$$
\lim _{u \in L_{G_{1}}(\Omega),\|u\|_{G_{1}} \rightarrow \infty} \frac{1}{\|u\|_{G_{1}}} \int_{\Omega} G_{1}(u) d x=\infty .
$$

Since $\|\cdot\|_{G}$ and $\|\cdot\|_{G_{1}}$ are equivalent on $L_{G_{1}}(\Omega)=L_{G}(\Omega)$, we have $k_{1}\|u\|_{G} \leq$ $\|u\|_{G_{1}} \leq k_{2}\|u\|_{G}$, for all $u \in L_{G}(\Omega)$ for some $k_{1}, k_{2}>0$. Moreover, since $G_{1} \sim G$, there are $L_{1}, M_{1}>0$ such that

$$
G_{1}\left(L_{1} t\right) \leq G(t), \quad \text { for all } t \in \mathbb{R}^{N} \text {, with }|t| \geq M_{1} .
$$

Since $G$ satisfies (G5'), $G^{*}$ and thus $H$ satisfy (G2'). As a consequence, $G_{1}$ also satisfies (G5'). Hence, from (2.39), there is $C_{1}>0$ such that $G(t) \geq$ $G_{1}\left(L_{1} t\right)-C_{1}$, for all $t \in \mathbb{R}^{N}$. We have, for $u \in L_{G}(\Omega)$,

$$
\begin{aligned}
\frac{1}{\|u\|_{G}} \int_{\Omega} G(u) d x & \geq \frac{1}{k_{2}\|u\|_{G_{1}}} \int_{\Omega}\left[G_{1}\left(L_{1} u\right)-C_{1}\right] d x \\
& \geq \frac{L_{1}}{k_{2}}\left[\frac{1}{\left\|L_{1} u\right\|_{G_{1}}} \int_{\Omega} G_{1}\left(L_{1} u\right) d x-C_{1}|\Omega|\right],
\end{aligned}
$$

where $|\Omega|$ is the measure of $\Omega$. Since $\|u\|_{G}$ is large if and only $\left\|L_{1} u\right\|_{G_{1}}$ is, this estimate and (2.38) imply (2.17).

Remark 2.9. Proposition 2.8 is an extension of Lemma 3.14 in [16] for $\mathrm{N}$ functions to the vector case of $G$-functions. Its alternate proof given here also fixes a seeming gap in the proof of Lemma 3.14 in [16]. In fact, in the second part of that proof in [16] (we use the notation of [16]) when $\bar{M}$ is assumed to 
have a $\Delta_{2}$ property for large value of $t$, we have $M(s t) \geq s g(s) M(t)$ only for $t \geq t_{0}$ and $s \geq K$. For $x \in \Omega_{u}$, we have $|u(x)| \geq t_{0}$ and thus

$$
M(|u(x)|)=M\left[\left(\|u\|_{(M), \Omega_{u}}-\varepsilon\right) \frac{|u(x)|}{\|u\|_{(M), \Omega_{u}}-\varepsilon}\right] .
$$

In order to apply the above equality on $M(s t)$, we need both $\|u\|_{(M), \Omega_{u}}-\varepsilon \geq k$ and $|u(x)| /\left(\|u\|_{(M), \Omega_{u}}-\varepsilon\right) \geq t_{0}$. The first inequality holds because $\|u\|_{(M), \Omega_{u}}>k$. However, the second inequality does not hold in general in $\Omega_{u}$. The reason is that although $|u(x)| \geq t_{0}$ by the definition of $\Omega_{u},\|u\|_{(M), \Omega_{u}}-\varepsilon$ can be large, which makes the fraction $|u(x)| /\left(\|u\|_{(M), \Omega_{u}}-\varepsilon\right)$ less than $t_{0}$.

\section{Equations and variational inequalities with anisotropic principal parts}

In this section, we consider precise settings for (1.1) and its weak formulations. We also prove general existence theorems and study some properties of the resolvent mapping. Assume $\Phi: \mathbb{R}^{N} \rightarrow[0, \infty)$ is a coercive $G$-function, i.e. $\Phi$ satisfies conditions (G1), (G2'), (G3), (G4), (G5'), (G6). As noted above, the conjugate $\Phi^{*}$ of $\Phi$ also satisfies these conditions. We also assume that both $\Phi$ and $\Phi^{*}$ satisfy $\Delta_{2}$ conditions at infinity in the sense of Definition 2.1. Assume $A$ is a Young function satisfying Trudinger's embedding theorem, i.e. (2.11)-(2.12) above. Therefore, we have (2.13) and (2.14). Let $\Phi_{0}$ be an $N$-function (cf. [1]) such that

$$
\Phi_{0} \ll A,
$$

(in the sense of Young functions, we refer to [40], [26], [1], [10] for definitions and properties of Young functions and $N$-functions). We know that the conjugate $\Phi_{0}^{*}$ of $\Phi_{0}$ is also an $N$-function. Furthermore, it follows that the $G$-function $G: \mathbb{R}^{N+1} \rightarrow[0, \infty]$

$$
G\left(u_{0}, u\right)=\Phi_{0}\left(u_{0}\right)+\Phi(u) \quad\left(u_{0} \in \mathbb{R}, u \in \mathbb{R}^{N}\right),
$$

also satisfies the conditions (G1), (G2'), (G3), (G4), (G5'), (G6). It is easy to prove that

$$
G^{*}\left(v_{0}, v\right)=\Phi_{0}^{*}\left(v_{0}\right)+\Phi^{*}(v), \quad \text { for all }\left(v_{0}, v\right) \in \mathbb{R}^{N+1} .
$$

For simplicity of calculations, we also assume here that

$\Phi_{0}$ and $\Phi_{0}^{*}$ satisfies $\Delta_{2}$ conditions at infinity.

It follows from (3.2)-(3.3) that $G$ and $G^{*}$ also satisfy $\Delta_{2}$ conditions at infinity. As a consequence (cf. e.g. [46] or [24]), $L_{G}$ and thus $W^{1} L_{G}, W_{0}^{1} L_{G}$ are reflexive and moreover $\left(L_{G}\right)^{*}=L_{G^{*}}$ under the usual pairing given by integrals. 
We study the boundary value problem (1.1) with the above setting. The weak formulation of (1.1) is

$$
\left\{\begin{array}{l}
\int_{\Omega} \nabla \Phi(\nabla u) \nabla v d x=\int_{\Omega} f(x, u) v d x, \text { for all } v \in X, \\
u \in X
\end{array}\right.
$$

where $X$ is an appropriate function space. Because of the growth condition of the principal term and the boundary condition in (1.1), a natural choice of $X$ is the anisotropic Orlicz-Sobolev space defined by $\Phi$,

$$
X=H^{0}(\Omega, \Phi)=W_{0}^{1} L_{G},
$$

where $G$ is given by (3.1)-(3.2). The appropriateness of this choice of function space is reflected in the following property, which means that the integral in the left hand side of (3.5) is defined for all $u, v \in X$.

Proposition 3.1. Assume $\Phi$ is differentiable in $\mathbb{R}^{N}$. Then

$$
\nabla \Phi(\nabla u) \nabla v \in L^{1}(\Omega), \quad \text { for all } u, v \in X .
$$

Proof. First, note that

$$
\Phi^{*}(\nabla \Phi(\xi)) \leq \Phi(2 \xi), \quad \text { for all } \xi \in \mathbb{R}^{N} .
$$

In fact, since $\Phi$ is differentiable, we have $\partial \Phi(\xi)=\{\nabla \Phi(\xi)\}$, for all $\xi \in \mathbb{R}^{N}$. Also (cf. e.g. [3]) $\nabla \Phi(\xi) \xi=\Phi(\xi)+\Phi^{*}(\nabla \Phi(\xi))$ and thus $\Phi(\xi) \leq \nabla \Phi(\xi) \xi \leq$ $\Phi(2 \xi)-\Phi(\xi)$. Hence, $\Phi^{*}(\nabla \Phi(\xi))=\nabla \Phi(\xi) \xi-\Phi(\xi) \leq \Phi(2 \xi)-2 \Phi(\xi) \leq \Phi(2 \xi)$. For almost every $x \in \Omega$, we have from Young's inequality,

$$
\begin{aligned}
|\nabla \Phi(\nabla u(x)) \nabla v(x)| & \leq \Phi(\nabla v(x))+\Phi^{*}(\nabla \Phi(\nabla u(x))) \\
& \leq \Phi(\nabla v(x))+\Phi(2 \nabla u(x)) .
\end{aligned}
$$

Since $\Phi$ satisfies a $\Delta_{2}$ condition at $\infty$, the integrals $\int_{\Omega} \Phi(2 \nabla u) d x$ and $\int_{\Omega} \Phi(\nabla v) d x$ are finite, i.e. $\Phi(\nabla v), \Phi(2 \nabla u) \in L^{1}(\Omega)$. (3.9) thus implies (3.7).

Let us consider the functional

$$
J: X \rightarrow[0, \infty], \quad J(u)=\int_{\Omega} \Phi(\nabla u) d x, \quad u \in X
$$

Since $\Phi$ is convex, so is $J$. Moreover, since $\Phi$ satisfies a $\Delta_{2}$ condition, $D(J)=X$. A standard application of Fatou's lemma shows that $J$ is lower semicontinuous on $X$. As a consequence, $J$ is locally Lipschitz on $X$. However, if $\Phi$ is not differentiable on $\mathbb{R}^{N}, J$ is not differentiable on $X$ in general. On the other hand, if $\Phi$ has some smoothness on $\mathbb{R}^{N}$ then $J$ inherits such property on $X$, which also shows the natural choice of $X$ as a suitable function space for problem (1.1). We have the following result about the derivative of $J$ on $X$. For a proper convex functional $f$ from a normed vector space $Z$ into $\mathbb{R} \cup\{\infty\}$, and for $u \in D(f)$, 
$h \in Z$, we use the usual notation $D f(u) h$ for the directional derivative of $f$ at $u$ in the direction $h$.

Proposition 3.2. (a) For any $u, h \in X$, the directional derivative of $J$ at $u$ in the direction of $h$ exists and is given by

$$
D J(u) h=\int_{\Omega} D \Phi(\nabla u(x)) \nabla h(x) d x
$$

(b) Assume $\Phi$ is Gâteaux differentiable on $\mathbb{R}^{N}$ and

$$
\Phi(\xi) \neq 0, \quad \text { for all } \xi \in \mathbb{R}^{N} \backslash\{0\}
$$

Then $J$ is Gâteaux differentiable on $X$ and $D J$ is given by (3.10).

(c) If $\Phi$ is of class $C^{1}$ on $\mathbb{R}^{N}$ and satisfies (3.11) then $J$ is of class $C^{1}$ on $X$ and

$$
\left\langle J^{\prime}(u), h\right\rangle=\int_{\Omega} \nabla \Phi(\nabla u(x)) \nabla h(x) d x
$$

Proof. We present the proof of (a) in detail and skip those of (b) and (c) since they are similar. Since $J$ is convex with $D(J)=X$, the directional derivative $D J(u) h$,

$$
D J(u) h=\lim _{t \rightarrow 0^{+}} \frac{J(u+t h)-J(u)}{t},
$$

exists in $[-\infty, \infty]$ for all $u, h \in X$. Let us prove that $D J(u) h$ is given by $(3.10)$ in our case. For $t>0$, we have

$$
\frac{1}{t}[J(u+t h)-J(u)]=\int_{\Omega} \frac{1}{t}[\Phi(\nabla u(x)+t h(x))-\Phi(\nabla u(x))] d x .
$$

Since $\Phi$ is convex with $D(\Phi)=\mathbb{R}^{N}$ (cf. assumption $\left(\mathrm{G} 5^{\prime}\right)$ ), the limit

$$
\lim _{t \rightarrow 0^{+}} \frac{1}{t}[\Phi(\nabla u(x)+t h(x))-\Phi(\nabla u(x))]=D \Phi(\nabla u(x))(\nabla h(x))
$$

exits in $[-\infty, \infty]$ for almost every $x \in \Omega$. The function $x \mapsto D \Phi(\nabla u(x))(\nabla h(x))$ is thus measurable on $\Omega$. Moreover (cf. Proposition 4.1 in [3]),

$$
\begin{aligned}
\Phi(\nabla u(x))-\Phi(\nabla u(x)-\nabla h(x)) & \leq D \Phi(\nabla u(x))(\nabla h(x)) \\
& \leq \Phi(\nabla u(x)+\nabla h(x))-\Phi(\nabla u(x)),
\end{aligned}
$$

for almost every $x \in \Omega$. 
Since the function $t \mapsto[\Phi(\nabla u(x)+t \nabla h(x))-\Phi(\nabla u(x))] / t$ is increasing on $(0, \infty)$, we have for $t \in(0,1), x \in \Omega$,

$$
\begin{aligned}
-\frac{1}{2} \Phi(2 \nabla u(x)) & -\frac{1}{2} \Phi(2 \nabla h(x)) \leq \Phi(\nabla u(x))-\Phi(\nabla u(x)-\nabla h(x)) \\
& \leq D \Phi(\nabla u(x)) \nabla h(x) \leq \inf _{s>0}\left\{\frac{1}{s}[\Phi(\nabla u(x)+s \nabla h(x))-\Phi(\nabla u(x))]\right\} \\
& \leq \frac{1}{t}[\Phi(\nabla u(x)+t \nabla h(x))-\Phi(\nabla u(x))] \\
& \leq \Phi(\nabla u(x)+\nabla h(x))-\Phi(\nabla u(x)) \leq \frac{1}{2} \Phi(2 \nabla u(x))+\frac{1}{2} \Phi(2 \nabla h(x)) .
\end{aligned}
$$

Consequently,

$$
\left|\frac{1}{t}[\Phi(\nabla u(x)+t \nabla h(x))-\Phi(\nabla u(x))]\right| \leq \frac{1}{2} \Phi(2 \nabla u(x))+\frac{1}{2} \Phi(2 \nabla h(x)),
$$

for all $t \in(0,1)$, almsot every $x \in \Omega$. Since $\Phi$ satisfies a $\Delta_{2}$ condition and $\nabla u, \nabla h \in L_{\Phi}(\Omega)$, the function in the right hand side of (3.14) belongs to $L^{1}(\Omega)$. From (3.12), (3.14), and the Lebesgue dominated convergence theorem, we get

$$
\begin{aligned}
D J(u) h & =\int_{\Omega} \lim _{t \rightarrow 0^{+}}\left\{\frac{1}{t}[\Phi(\nabla u(x)+t \nabla h(x))-\Phi(\nabla u(x))]\right\} d x \\
& =\int_{\Omega} D \Phi(\nabla u(x)) \nabla h(x) d x .
\end{aligned}
$$

REMARK 3.3. Note that for any $G$-function $\Phi$ satisfying (G1), (G2'), (G3), (G4), (G5'), by using the construction in Proposition 2.8, we see that there always exists a $G$-function $\Phi_{1}$ that satisfies these conditions and also (3.11). Therefore in the sequel we assume $\Phi$ satisfies (3.11).

In what follows, we are interested in nonsmooth $G$-functions $\Phi$, that is $\Phi$ is assumed only to satisfy (G1), (G2'), (G3), (G4), (G5'), (G6) and thus may not be differentiable in $\mathbb{R}^{N}$. If $\mathcal{F}$ is given by

$$
\langle\mathcal{F}(u), v\rangle=\int_{\Omega} f(x, u) v d x .
$$

then under certain growth condition of $f, \mathcal{F}(u)$ belongs to $X^{*}$ for any $u \in X$. Equation (3.5) now becomes

$$
D J(u)(v)=\langle\mathcal{F}(u), v\rangle, \quad \text { for all } v \in X .
$$

In case $J$ is not differentiable, thanks to its convexity, we can consider instead of equation (3.16), the inclusion $\partial J(u) \ni \mathcal{F}(u)$ or equivalently,

$$
\partial J(u)-\mathcal{F}(u) \ni 0 \text { in } X^{*} .
$$

Inclusion (3.17) is equivalent to the variational inequality

$$
\left\{\begin{array}{l}
J(v)-J(u)-\langle\mathcal{F}(u), v-u\rangle \geq 0, \quad \text { for all } v \in X, \\
u \in X
\end{array}\right.
$$


In what follows, we study instead of (3.18) a more general variational inequality:

$$
\left\{\begin{array}{l}
J(v)-J(u)-\langle\mathcal{F}(u), v-u\rangle \geq 0, \quad \text { for all } v \in K, \\
u \in K,
\end{array}\right.
$$

where $K$ is a closed convex subset of $X$. We have the following abstract existence result for (3.19).

Theorem 3.4. Assume

$$
\mathcal{F}: X \rightarrow X^{*} \text { is a pseudomonotone mapping, }
$$

and there are $u_{0} \in X$ and $R>\left\|u_{0}\right\|$ such that

$$
K \cap B_{R}(0) \neq \emptyset,
$$

and

$$
\left\langle\mathcal{F}(u), u-u_{0}\right\rangle+J(u)>J\left(u_{0}\right),
$$

for all $u \in K$ with $\|u\|=R$. Then the variational inequality (3.19) has solutions.

Proof. Theorem 3.4 is a direct consequence of Corollary 2.6 in [33]. In fact, since $\Phi$ satisfies a $\Delta_{2}$ condition at $\infty$, we see that $J$ is bounded on bounded sets of $X$ and thus locally Lipschitz on $X$. It follows (cf. e.g. Theorem 4.2 in [3]) that $\partial J(u) \neq \emptyset$, for all $u \in X$. Furthermore,

$$
D\left(\partial\left(J+I_{K}\right)\right)=K .
$$

In fact, since $\left(J+I_{K}\right)(u)=\infty$ if $u \notin K$, we have $D\left(\partial\left(J+I_{K}\right)\right) \subset K$. If $u \in K$ then for $l \in \partial J(u)$, we have for any $v \in X$,

$$
\left(J+I_{K}\right)(v)-\left(J+I_{K}\right)(u) \geq J(v)-J(u) \geq\langle l, v-u\rangle .
$$

This shows that $l \in \partial\left(J+I_{K}\right)(u)$, i.e. $\partial\left(J+I_{K}\right)(u) \neq \emptyset$, and thus $u \in D(\partial(J+$ $\left.I_{K}\right)$ ), proving (3.23). Note that $X$ is reflexive since both $G$ and $G^{*}$ satisfy $\Delta_{2}$ conditions at infinity and thus Corollary 2.6 in [33] is applicable in our problem here. Also, (3.21)-(3.23) imply that the conditions in Corollary 2.6 of [33] are satisfied with $\psi=J, \phi=J+I_{K}, L=0$, and $B=-\mathcal{F}$. According to that result, inequality (3.19) has solutions.

As a consequence of Theorem 3.4, we have the following existence result.

Corollary 3.5. Assume $\mathcal{F}$ is a pseudomonotone mapping such that

$$
\left\langle\mathcal{F}(u), u-u_{0}\right\rangle \geq-\alpha_{1}\|u\|-\beta_{1}, \quad \text { for all } u \in K,
$$

for some $\alpha_{1}, \beta_{1} \geq 0$. Then (3.19) has solutions. 
ProOF. It follows from (3.24) that

$$
\frac{J(u)+\left\langle\mathcal{F}(u), u-u_{0}\right\rangle}{\|u\|} \geq \frac{J(u)}{\|u\|}-\alpha_{1}-\frac{\beta_{1}}{\|u\|},
$$

for all $u \in K \backslash\{0\}$. From (2.17) in Proposition 2.7, we see that

$$
\lim _{\|u\| \rightarrow \infty, u \in K} \frac{J(u)+\left\langle\mathcal{F}(u), u-u_{0}\right\rangle}{\|u\|}=\infty
$$

which implies (3.22).

It is clear that $\mathcal{F}(u)=L \in X^{*}$ satisfies (3.24), thus the set $S_{K}(L)$ of solutions to the variational inequality

$$
\left\{\begin{array}{l}
J(v)-J(u) \geq\langle L, v-u\rangle, \quad \text { for all } v \in K \\
u \in K
\end{array}\right.
$$

or equivalently, of the inclusion $\partial J(u) \ni L$ is nonempty.

REMARK 3.6. Note that in this particular case where $f=L \in X^{*},(3.25)$ is equivalent to the minimization theorem

$$
u \in K: J(u)-\langle L, u\rangle=\min _{v \in K}[J(v)-\langle L, v\rangle] .
$$

From the coercivity property of $J$ given in Proposition 2.7 and its lower semicontinuity with respect to the weak* topology of $X$ (cf. Theorem 2.1, Chapter 8, [11]), we obtain the existence of solutions of (3.26) and thus of (3.25) for any weakly* closed and convex subset $K$ of $X$, with a $\Delta_{2}$ condition assumed only on $G^{*}$ but not necessarily on $G$.

The mapping $S_{K}: L \mapsto S_{K}(L)$ is resolvent (mapping) solution of (3.25). In the particular case where $K=X$ and $\Phi$ is smooth, $S_{X}$ is the same as the inverse operator $[-\operatorname{div}(\nabla \Phi(\nabla(\cdot)))]^{-1}$. Some properties of $S_{K}$ are given in the following result.

THEOREM 3.7. (a) $u \in S_{K}(L)$ if and only if $u$ is a minimizer of the functional $J-L$ on $K$.

(b) For any $L \in X^{*}, S_{K}(L)$ is a nonempty closed convex and bounded subset of $K$. If $\Phi$ is strictly convex then $S_{K}(L)$ is a singleton.

(c) The mapping $S_{K}: X^{*} \rightarrow 2^{X}$ is a bounded and strong-weak upper semicontinuous, i.e. upper semicontinuous from $X^{*}$ with the strong topology to $2^{X}$ with the weak topology on $X$.

ProOF. The proof of (a) is straightforward.

(b) As noted above, $S_{K}(L) \neq \emptyset$. The convexity, boundedness, and closedness of $S_{K}(L)$ follows directly from the convexity, coercivity (cf. Proposition 2.7), and lower semicontinuity of $J$. When $\Phi$ is strictly convex on $\mathbb{R}^{N}$, it is straightforward 
to show that $J$ is also strictly convex on $X$. Therefore, $J-L$ has a unique minimizer in $K$, which is the unique element of $S_{K}(L)$.

(c) To prove the boundedness of $S_{K}$, let $W$ be a bounded set in $X^{*}$ and $M=\sup \left\{\|L\|_{*}: L \in W\right\}(<\infty)$. Let $u_{0}$ be a fixed element of $K$ and put $M_{0}=\left|J\left(u_{0}\right)-\left\langle L, u_{0}\right\rangle\right|$. From Proposition 2.7, there exists $\rho>1$ such that $J(u) /\|u\|>M+M_{0}$ for all $u \in X$ with $\|u\|>\rho$. Let $L \in W$ and $u \in S_{K}(L)$. We have $J(u)-\langle L, u\rangle \leq J\left(u_{0}\right)-\left\langle L, u_{0}\right\rangle \leq M_{0}$ and thus

$$
\frac{J(u)}{\|u\|} \leq \frac{|\langle L, u\rangle|}{\|u\|}+\frac{M_{0}}{\|u\|} \leq\|L\|_{*}+M_{0} \leq M+M_{0}
$$

if $\|u\|>\rho$. This implies from the choice of $\rho$ that $\|u\| \leq \rho$. Hence $\bigcup\left\{S_{K}(L)\right.$ : $L \in W\} \subset \overline{B_{\rho}(0)}$, proving that $S_{K}$ is a bounded mapping.

Let $L_{0} \in X^{*}$. To prove the strong-weak upper semicontinuity of $S_{K}$ at $L_{0}$, we assume by contradiction that there exists a weakly open set $W$ in $X$ and a sequence $\left\{L_{n}\right\} \subset X^{*}$ such that

$$
L_{n} \rightarrow L_{0} \text { in } X^{*}, \quad S\left(L_{0}\right) \subset W, \quad \text { but } \quad S\left(L_{n}\right) \not \subset W, \quad \text { for all } n \in \mathbb{N} .
$$

Therefore, for each $n \in \mathbb{N}$, there exists

$$
u_{n} \in S\left(L_{n}\right) \backslash W .
$$

Since $\left\{L_{n}\right\}$ is bounded, $\left\{u_{n}\right\}$ is a bounded sequence in $K$. Because $X$ is reflexive and $K$ is weakly closed in $X$, by passing to a subsequence if necessary we can assume that

$$
u_{n} \rightarrow u_{0} \in K .
$$

The weak lower semicontinuity of $J$ on $X$ implies that

$$
J\left(u_{0}\right) \leq \liminf J\left(u_{n}\right) .
$$

On the other hand, (3.27) and (3.29) implies that

$$
\left\langle L_{n}, u_{n}\right\rangle \rightarrow\left\langle L_{0}, u_{0}\right\rangle .
$$

Let $v \in K$. For any $n \in \mathbb{N}$, we have $J(v)-\left\langle L_{n}, v\right\rangle \geq J\left(u_{n}\right)-\left\langle L_{n}, u_{n}\right\rangle$. Letting $n \rightarrow \infty$ and taking into account (3.30) and (3.31), we have

$$
\begin{aligned}
J(v)-\langle L, v\rangle & =\lim \left[J(v)-\left\langle L_{n}, v\right\rangle\right] \geq \liminf \left[J\left(u_{n}\right)-\left\langle L_{n}, u_{n}\right\rangle\right] \\
& =\liminf J\left(u_{n}\right)-\lim \left\langle L_{n}, u_{n}\right\rangle \geq J\left(u_{0}\right)-\left\langle L_{0}, u_{0}\right\rangle .
\end{aligned}
$$

This shows that $u_{0} \in S_{K}\left(L_{0}\right)$. From (3.29), we see that $u_{n} \in W$ for all $n$ sufficiently large, contradicting (3.28). This contradiction proves the strongweak upper semicontinuity of $S_{K}$.

When $K=X$ and $\Phi$ is strictly convex, the continuity of $S_{X}$ in Theorem 3.7(c) can be strengthened as follows. 
TheOREM 3.8. Assume $K=X$ and $\Phi$ is strictly convex on $\mathbb{R}^{N}$. Then for each $L \in X^{*}, S_{X}(L)=\left\{u_{L}\right\}$ and the mapping $S_{X}: L \mapsto u_{L}$ is continuous from $X^{*}$ to $X$.

The proof of this theorem is based on the following lemma about a compactness property of $\partial J$ on $X$ which seems to have some interest itself.

Lemma 3.9. Let $\left\{u_{n}\right\}$ be a bounded sequence in $X,\left\{\xi_{n}\right\}$ a sequence in $X^{*}$, $u \in X$, and $\xi \in X^{*}$ such that $\xi \in \partial J(u), \xi_{n} \in \partial J\left(u_{n}\right)$, for all $n \in \mathbb{N}$. Furthermore,

$$
\left\langle\xi_{n}-\xi, u_{n}-u\right\rangle \rightarrow 0 \text { as } n \rightarrow \infty
$$

then $u_{n} \rightarrow u$ in $X$.

Proof. Assume $u, \xi, u_{n}, \xi_{n}$ be as in the assumptions of Lemma 3.9. Note that $X$ can be identified with a closed subspace $\tilde{X}$ of $L_{\Phi}(\Omega)$ :

$$
\widetilde{X}=\left\{\left(w_{1}, \ldots, w_{N}\right) \in L_{\Phi}(\Omega): \exists u \in W_{0}^{1,1}(\Omega): \partial_{j} u=w_{j} \text { a.e. on } \Omega\right\} .
$$

Therefore, from Theorem 3.3 in [25], we have, for any $w \in X$,

$$
\partial J(w) \subset \int_{\Omega} \partial \Phi(\nabla w(x)) \nabla(\cdot) d x,
$$

i.e. for any $\eta \in \partial J(w)$, there exists a measurable function $\widehat{\eta}: \Omega \rightarrow \mathbb{R}^{N}$ such that

$$
\widehat{\eta}(x) \in \partial \Phi(\nabla w(x)) \text { for a.e. } x \in \Omega,
$$

$\widehat{\eta} \nabla v \in L^{1}(\Omega)$, and

$$
\langle\eta, v\rangle=\int_{\Omega} \widehat{\eta}(x) \nabla v(x) d x, \quad \text { for all } v \in X .
$$

Let $\widehat{\xi}_{n}, \widehat{\xi}$ correspond to $\xi_{n}, \xi$ and $u_{n}, u$ as in (3.33)-(3.34). From (3.33), we have

$$
\widehat{\eta}(x) \nabla w(x)=\Phi(\nabla w(x))+\Phi^{*}(\widehat{\eta}(x)),
$$

for almost every $x \in \Omega$. Hence, from (3.34),

$$
\infty>\langle\eta, w\rangle=\int_{\Omega} \widehat{\eta}(x) \nabla w(x) d x=\int_{\Omega} \Phi(\nabla w(x)) d x+\int_{\Omega} \Phi^{*}(\widehat{\eta}(x)) d x .
$$

This implies that

$$
\Phi^{*}(\widehat{\eta}) \in L^{1}(\Omega), \quad \text { i.e. } \widehat{\eta} \in L_{\Phi^{*}}(\Omega) .
$$

On the other hand, since $\Phi$ is strictly convex, $\partial \Phi$ is strictly monotone on $\mathbb{R}^{N}$, that is, for all $z, z^{\prime} \in \mathbb{R}^{N}, z \neq z^{\prime}$, all $\widehat{z} \in \partial \Phi(z), \widehat{z^{\prime}} \in \partial \Phi\left(z^{\prime}\right)$, we have

$$
\left\langle\widehat{z}-\widehat{z^{\prime}}, z-z^{\prime}\right\rangle>0 \text {. }
$$

Therefore,

$$
\left\langle\widehat{\xi}_{n}(x)-\widehat{\xi}(x), \nabla u_{n}(x)-\nabla u(x)\right\rangle \geq 0, \quad \text { for a.e. } x \in \Omega, \text { all } n \in \mathbb{N} .
$$


Since $\left\langle\xi_{n}, v\right\rangle=\int_{\Omega} \widehat{\xi}_{n} \nabla v d x$ and $\langle\xi, v\rangle=\int_{\Omega} \widehat{\xi} \nabla v d x$, we have

$$
\left\langle\xi_{n}-\xi, u_{n}-u\right\rangle=\int_{\Omega}\left(\widehat{\xi}_{n}-\widehat{\xi}\right)\left(\nabla u_{n}-\nabla u\right) d x .
$$

(3.37) and (3.32) imply that

$$
\left(\widehat{\xi}_{n}-\widehat{\xi}\right)\left(\nabla u_{n}-\nabla u\right) \rightarrow 0 \quad \text { in } L^{1}(\Omega) .
$$

We prove that for any $\varepsilon>0$, there exists $\delta \in(0, \varepsilon)$ such that for all $H \subset \Omega$ measurable with $|H|<\delta(|H|$ is the Lebesgue measure of $H)$, we have

$$
\int_{H} \Phi\left(\nabla u_{n}\right) d x<\varepsilon, \quad \text { for all } n \in \mathbb{N} .
$$

In fact, given $\varepsilon>0$, it follows from (3.38) that there exists $n_{1} \in \mathbb{N}$ such that

$$
\int_{\Omega}\left(\widehat{\xi}_{n}-\widehat{\xi}\right)\left(\nabla u_{n}-\nabla u\right) d x<\frac{\varepsilon}{4}, \quad \text { for all } n \geq n_{1} .
$$

Together with (3.37), this implies that

$$
\int_{H}\left(\widehat{\xi}_{n}-\widehat{\xi}\right)\left(\nabla u_{n}-\nabla u\right) d x<\frac{\varepsilon}{4}, \quad \text { for all } n \geq n_{1},
$$

for any measurable subset $H$ of $\Omega$. Therefore,

$$
\begin{aligned}
\int_{H} \widehat{\xi}_{n} \nabla u_{n} d x & <\frac{\varepsilon}{4}+\int_{H} \widehat{\xi}_{n} \nabla u d x+\int_{H} \widehat{\xi} \nabla u_{n} d x-\int_{H} \widehat{\xi} \nabla u d x \\
& \leq \frac{\varepsilon}{4}+\int_{H} \widehat{\xi}_{n} \nabla u d x+\int_{H} \widehat{\xi} \nabla u_{n} d x .
\end{aligned}
$$

In the next step, we present some inequalities that are useful in the next calculations. First, basic properties of convex functions give us

$$
\Phi(z) \leq \zeta z \leq \Phi(2 z),
$$

and

$$
\Phi^{*}(\zeta) \leq \Phi(2 z),
$$

for all $z \in \mathbb{R}^{N}$, all $\zeta \in \partial \Phi(z)$. On the other hand, we have from Young's inequality that for all $\rho \in(0, \infty)$, all $z_{1}, z_{2} \in \mathbb{R}^{N}$,

$$
\left|z_{1} z_{2}\right| \leq \Phi\left(\rho^{-1} z_{1}\right)+\Phi^{*}\left(\rho z_{2}\right) .
$$

Since $\Phi$ satisfies a $\Delta_{2}$ condition, there are $K, b \geq 0$ such that

$$
\Phi(2 z) \leq K \Phi(z)+b, \quad \text { for all } z \in \mathbb{R}^{N} .
$$

For any $x \in \Omega, \rho \in(0,1)$, we have

$$
\begin{aligned}
\left|\widehat{\xi}_{n}(x) \nabla u(x)\right| & \leq \Phi\left(\rho^{-1} \nabla u(x)\right)+\Phi^{*}\left(\rho \widehat{\xi}_{n}(x)\right) \\
& \leq \Phi\left(\rho^{-1} \nabla u(x)\right)+\rho \Phi^{*}\left(\widehat{\xi}_{n}(x)\right) .
\end{aligned}
$$


Similarly,

$$
\begin{aligned}
\left|\widehat{\xi}(x) \nabla u_{n}(x)\right| & \leq \Phi\left(\rho \nabla u_{n}(x)\right)+\Phi^{*}\left(\rho^{-1} \widehat{\xi}(x)\right) \\
& \leq \rho \Phi\left(\nabla u_{n}(x)\right)+\Phi^{*}\left(\rho^{-1} \widehat{\xi}(x)\right) .
\end{aligned}
$$

Also, since $\widehat{\xi}_{n}(x) \in \partial \Phi\left(\nabla u_{n}(x)\right)$ for almost every $x \in \Omega$, we have from (3.44) and (3.42) that for almost every $x \in \Omega$,

$$
\Phi^{*}\left(\widehat{\xi}_{n}(x)\right) \leq \Phi\left(2 \nabla u_{n}(x)\right) \leq K \Phi\left(\nabla u_{n}(x)\right)+b .
$$

Combining (3.40), (3.41), (3.45), (3.42), (3.47), (3.46) and simplifying the resulted estimate, we get

$$
\begin{aligned}
{[1-\rho(K+1)] \int_{H} \Phi\left(\nabla u_{n}\right) d x } & <\frac{\varepsilon}{4}+\left[\rho b+b^{*}(\rho)\right]|H| \\
+ & \int_{H} \Phi\left(\rho^{-1} \nabla u\right) d x+K^{*}(\rho) \int_{H} \Phi(2 \nabla u) d x .
\end{aligned}
$$

Choose $\rho=[2(K+1)]^{-1}$. Since $\Phi$ satisfies a $\Delta_{2}$ condition and $\nabla u \in L_{\Phi}$, we have $\Phi\left(\rho^{-1} \nabla u\right), \Phi(2 \nabla u) \in L^{1}(\Omega)$. Hence, there are $\delta_{1}, \delta_{2}>0$ such that if $|H|<\delta_{1}$ then

$$
\int_{H} \Phi\left(\rho^{-1} \nabla u\right)<\frac{\varepsilon}{12}
$$

and, if $|H|<\delta_{2}$, then

$$
\int_{H} \Phi(2 \nabla u) d x<\frac{\varepsilon}{12 K^{*}\left([2(K+1)]^{-1}\right)} .
$$

Letting

$$
\delta=\min \left\{\delta_{1}, \delta_{2}, \frac{\varepsilon}{12\left(b[2(K+1)]^{-1}+b^{*}\left([2(K+1)]^{-1}\right)\right)}\right\},
$$

we see that if $H \subset \Omega$ measurable and $|H|<\delta$ then the last three terms in the right hand side of (3.48) is less than $\varepsilon / 12$ and thus $\int_{H} \Phi\left(\nabla u_{n}\right) d x<\varepsilon$. (3.39) is proved.

Next, we prove that

$$
\nabla u_{n} \rightarrow \nabla u \quad \text { a.e. in } \Omega \text {. }
$$

From (3.38), there exist a subsequence $\left\{u_{n_{k}}\right\}$ of $\left\{u_{n}\right\}$ and a subset $U$ of $\Omega$ of measure zero such that for all $x \in \Omega \backslash U, \widehat{\xi}_{n}(x) \in \partial \Phi\left(\nabla u_{n}(x)\right), \widehat{\xi}(x) \in \partial \Phi(\nabla u(x))$, for all $n \in \mathbb{N}$ and

$$
\left.\left(\widehat{\xi}_{n_{k}}\right)(x)-\widehat{\xi}(x)\right)\left(\nabla u_{n_{k}}(x)-\nabla u(x)\right) \rightarrow 0 \quad \text { as } k \rightarrow \infty .
$$

In particular, for all $x \in \Omega \backslash U$, there is $\alpha_{0} \geq 0$ (which may depend on $x$ ) such that $\left.\left(\widehat{\xi}_{n_{k}}\right)(x)-\widehat{\xi}(x)\right)\left(\nabla u_{n_{k}}(x)-\nabla u(x)\right) \leq \alpha_{0}$, for all $k \in \mathbb{N}$. Using again 
the estimates in (3.40), (3.41), (3.45), (3.42), (3.47), and (3.46), we see that for all $x \in \Omega \backslash U$, all $k \in \mathbb{N}$,

$[1-\rho(K+1)] \Phi\left(\nabla u_{n_{k}}(x)\right) \leq \alpha_{0}+\rho b+b^{*}(\rho)+\Phi\left(\rho^{-1} \nabla u(x)\right)+K^{*}(\rho) \Phi(2 \nabla u(x))$.

By choosing $\rho=[2(K+1)]^{-1}$, we see that $\left\{\Phi\left(\nabla u_{n_{k}}(x)\right)\right\}$ is a bounded sequence for all $x \in \Omega \backslash U$. From (G2') (or even (G2)), this means that $\left\{\nabla u_{n_{k}}(x)\right\}$ is a bounded sequence in $\mathbb{R}^{N}$, which together with (3.47), implies that $\left\{\Phi^{*}\left(\widehat{\xi}_{n_{k}}(x)\right)\right\}$ and thus $\left\{\widehat{\xi}_{n_{k}}(x)\right\}$ are bounded sequences (for all $x \in \Omega \backslash U$ ).

For each $x \in \Omega \backslash U$, by passing to subsequences (that may as well depend on $x$ ), we can assume for simplicity of notation that

$$
\begin{aligned}
\nabla u_{n_{k}}(x) \rightarrow w_{0} & =w_{0}(x) \quad \text { in } \mathbb{R}^{N}, \\
\widehat{\xi}_{n_{k}}(x) \rightarrow \widehat{\xi}_{0} & =\widehat{\xi}_{0}(x) \quad \text { in } \mathbb{R}^{N} .
\end{aligned}
$$

Since $\widehat{\xi}_{n_{k}}(x) \in \partial \Phi\left(\nabla u_{n_{k}}(x)\right)$, for all $k \in \mathbb{N}$, by the upper semicontinuity of the mapping $\partial \Phi: \mathbb{R}^{N} \rightarrow 2^{\mathbb{R}^{N}}, w \mapsto \partial \Phi(w)$, we see that $\widehat{\xi}_{0} \in \partial \Phi\left(w_{0}\right)$. Let $k \rightarrow \infty$ in (3.50), we see that $\left(\widehat{\xi}_{0}-\widehat{\xi}(x)\right)\left(w_{0}-\nabla u(x)\right)=0$. Due to the strict monotonicity of $\partial \Phi$, we conclude that $\nabla u(x)=w_{0}$, i.e. $\nabla u_{n_{k}}(x) \rightarrow \nabla u(x)$. Since this procedure holds for any subsequence of $\left\{\nabla u_{n}(x)\right\}$, we obtain (3.49). From (3.49) and the continuity of $\Phi$ in $\mathbb{R}^{N}$, we get

$$
\Phi\left(\nabla u_{n}-\nabla u\right) \rightarrow 0 \quad \text { a.e. in } \Omega .
$$

Let us prove that the sequence $\left\{\Phi\left(\nabla u_{n}-\nabla u\right)\right\}$ is uniformly integrable on $\Omega$. In fact, for all $n \in \mathbb{N}$ and almost every $x \in \Omega$,

$$
\begin{aligned}
\Phi\left(\nabla u_{n}(x)-\nabla u(x)\right) & \leq \frac{1}{2} \Phi\left(2 \nabla u_{n}(x)\right)+\frac{1}{2} \Phi(2 \nabla u(x)) \\
& \leq \frac{K}{2} \Phi\left(\nabla u_{n}(x)\right)+\frac{b}{2}+\frac{1}{2} \Phi(2 \nabla u(x)) .
\end{aligned}
$$

Hence for all $H \subset \Omega$ measurable,

$$
\int_{H} \Phi\left(\nabla u_{n}-\nabla u\right) d x \leq \frac{K}{2} \int_{H} \Phi\left(\nabla u_{n}\right) d x+\frac{b|H|}{2}+\frac{1}{2} \int_{H} \Phi(2 \nabla u) d x .
$$

Given $\varepsilon>0$. It follows from (3.39) that there exists $\delta_{3}>0$ such that $\int_{H} \Phi\left(\nabla u_{n}\right) d x$ $<2 \varepsilon /(3 K)$ whenever $H$ is a measurable subset of $\Omega$ with $|H|<\delta_{3}$. On the other hand, since $\Phi(2 \nabla u) \in L^{1}(\Omega)$, there is $\delta_{4}>0$ such that $\int_{H} \Phi(2 \nabla u) d x<2 \varepsilon / 3$ if $|H|<\delta_{4}$. Choosing $\delta=\min \left\{\delta_{3}, \delta_{4}, 2 \varepsilon /(3 b)\right\}$, we see from (3.54) that if $H \subset \Omega$ measurable and $|H|<\delta$ then $\int_{H} \Phi\left(\nabla u_{n}-\nabla u\right) d x<\varepsilon$ for all $n \in \mathbb{N}$.

According to Vitali's convergence theorem (cf. e.g. [23]), together with (3.53), this uniform integrability of $\left\{\Phi\left(\nabla u_{n}-\nabla u\right)\right\}$ shows that $\Phi\left(\nabla u_{n}-\nabla u\right) \rightarrow 0$ in $L^{1}(\Omega)$. Since $\Phi$ satisfies a $\Delta_{2}$ condition at $\infty$, the norm and modular convergences are equivalent in $L_{\Phi}(\Omega)$. Hence $\left\|\nabla u_{n}-\nabla u\right\|_{L_{\Phi}} \rightarrow 0$, i.e. $u_{n} \rightarrow u$ in $X$. $\square$ 
Corollary 3.10. If $\Phi$ is strictly convex in $\mathbb{R}^{N}$ then $\partial J: X \rightarrow 2^{X^{*}}$ is of class $(\mathrm{S})_{+}$in the following sense: If $u_{n} \rightarrow u$ in $X$ and there exists $\xi_{n} \in \partial J\left(u_{n}\right)$ such that $\lim \sup \left\langle\xi_{n}, u_{n}-u\right\rangle \leq 0$, then $u_{n} \rightarrow u$ in $X$.

ProOF. Let $\xi \in \partial J(u)$. We have $\left\langle\xi, u_{n}-u\right\rangle \rightarrow 0$ and thus $0 \leq \liminf \left\langle\xi_{n}-\xi, u_{n}-u\right\rangle \leq \lim \sup \left\langle\xi_{n}-\xi, u_{n}-u\right\rangle=\lim \sup \left\langle\xi_{n}, u_{n}-u\right\rangle \leq 0$. This shows that $\lim \left\langle\xi_{n}-\xi, u_{n}-u\right\rangle=0$. Also, since $\left\{u_{n}\right\}$ is weakly convergent, it is bounded. According to Lemma (3.9), we have $u_{n} \rightarrow u$ in $X$.

REMARK 3.11. In the case of modulars given by anisotropic convex functions as considered above, Corollary 3.10 improves Theorem 3.8 in [13] in the sense that we assume here the strict convexity of $\Phi$ instead of uniform convexity conditions as in [13]. Also, we follow here a different approach to prove Corollary 3.10.

We are now ready to prove Theorem 3.8 .

Proof Proof of Theorem 3.8. Since $\Phi$ is strictly convex on $\mathbb{R}^{N}, J$ is strictly convex on $X$. The uniqueness of the solution of (3.25) follows from the strict convexity of $J-L$. Let $\left\{L_{n}\right\} \subset X^{*}, L_{n} \rightarrow L_{0}$ in $X^{*}$. Let us prove that $u_{n} \rightarrow u_{0}$ in $X$ where $\left\{u_{n}\right\}=S_{X}\left(L_{n}\right), n \in \mathbb{N} \cup\{0\}$. In fact, from Theorem 3.7(c), we know that $u_{n} \rightarrow u_{0}$ in $X$. As above, we have $L_{n} \in \partial J\left(u_{n}\right)$, for all $n \in \mathbb{N}$ and also $u_{0} \in S_{X}\left(L_{0}\right)$, i.e. $L_{0} \in \partial J\left(u_{0}\right)$. Since $L_{n} \rightarrow L_{0}$ in $X^{*}$ and $u_{n} \rightarrow u_{0}$ in $X$, we get $\left\langle L_{n}-L_{0}, u_{n}-u_{0}\right\rangle \rightarrow 0$. It now follows from Lemma 3.9 that $u_{n} \rightarrow u$ in $X$. $\square$

REMARK 3.12. (a) Since we are interested here in variational inequalities on any closed and convex subset $K$ of $X$, the $\Delta_{2}$ conditions on both $G$ and $G^{*}$ are assumed here in order to apply the abstract existence results in [33] (Theorem 2.2 and Corollary 2.6, [33]) for the solvability of inclusions and variational inequalities in reflexive Banach spaces. $\Delta_{2}$ conditions are assumed on both $G$ and $G^{*}$ to guarantee the reflexivity of $X$ and thus the applicability of the above abstract theorems. As a consequence, we obtain in Theorem 3.4 and Corollary 3.5 the existence of solutions of inequalities in which the lower order terms (or right-hand-side terms) can be given by general pseudomonotone operators of $u$.

Another reason for such assumption about $\Delta_{2}$ conditions here is that we study, in addition to the existence of solutions of variational inequality (3.19) and inclusion (3.17), properties of their solutions and their related mappings as well. For example, we need a $\Delta_{2}$ condition on $G^{*}$ in the proof of Proposition 2.7 about a coercivity property of $J$ and a $\Delta_{2}$ condition on $G$ to get the $(\mathrm{S})_{+}$property of $\partial J$ in Corollary 3.10 .

(b) Compared to the papers [17] and [22], we note that in those papers the function spaces are general anisotropic Orlicz-Musielak spaces, that is, the 
generating $N$-functions depend also on $x$. It was not assumed in [17] any $\Delta_{2}$ condition on the $N$-function $M$ nor on its conjugate $M^{*}$ as we assume here. The right hand side $f$ was assumed in [17] to be a function on $\Omega$ which was the divergence of a vector field $F$ in $E_{M^{*}}$ which, roughly speaking, corresponds to the case $f=L \in X^{*}$ here. It was proved in [22] the existence and uniqueness of renormalized and, in particular cases, of weak solutions of the inclusion

$$
\beta(x, u)-\operatorname{div}(a(x, \nabla u)+F(u)) \ni f \text { in } \Omega,
$$

where $\beta$ is maximal monotone in the second variable without any further growth condition, $F$ is locally Lipschitz, and $f$ belongs to $L^{d}(\Omega)$ in the case of weak solutions ( $d$ is the dimension of $\Omega$ ). The problem was studied in a general OrliczMusielak space where the conjugate of the generating function satisfied a $\Delta_{2}$ condition. The existence results in the above works were based on truncation methods together with Minty-Browder type techniques.

Here, we get the existence of solutions of variational inequalities in anisotropic Orlicz-Sobolev spaces (generated by $G$-functions that do not depend on $x$ ) by a combination of variational and monotonicity approaches based on an abstract existence theorem. Compared to [17] and [22], the different approaches lead to existence results of different natures. As noted in (a), we assumed in Theorem 3.4 $\Delta_{2}$ conditions on both $G$ and $G^{*}$, but we get the existence of solutions of variational inequalities rather than of equations, with right hand sides of different nature (pseudomonotone terms that may depend on $u$ ). In the illustrative example in Section 5, the signed term $h$ is assumed to have subcritical growth but is not necessarily monotone in $u$, while the "sublinear" term $g$ depends on $u$ and has no monotone property in general. Therefore, even in the case of equation (i.e. $K=X$, in problem with $\Delta_{2}$ conditions), that particular example seems not to be completely covered by the theories in [17] and [22]. Furthermore, we are interested here in some other properties of solutions and of their related mappings.

\section{Fixed point formulation and topological degree}

By using the resolvent mapping $S_{K}$, we can write the variational inequality (3.19) equivalently as the fixed point inclusion

$$
u \in X: u \in S_{K}(\mathcal{F}(u)) .
$$

In cases where $\mathcal{F}$ has some compactness properties, the existence and other properties of solutions of (4.1) can be studied by fixed point or topological methods. An example of such situations is presented here.

Assume $f: \Omega \times \mathbb{R} \rightarrow \mathbb{R}$ is a Carathéodory function such that

$$
|f(x, u)| \leq B_{0}(x)+C_{0}\left[\left(\Phi_{0}^{*}\right)^{-1} \Phi_{0}\right](|u|),
$$


for almost every $x \in \Omega$, all $u \in \mathbb{R}$, where $B_{0} \in L_{\Phi_{0}^{*}}$ and $C_{0}>0$. Note that $\Phi_{0}$ is given in (3.1)-(3.2) and condition (4.2) is an extension of the classical subcritical growth condition to our problem with anisotropic principal term.

From (3.1), the embedding $i=i_{\Phi_{0}}: X \hookrightarrow L_{\Phi_{0}}$ is compact, and so is its adjoint $i^{*}: L_{\Phi_{0}^{*}}\left(=\left(L_{\Phi_{0}}\right)^{*}\right) \hookrightarrow X^{*}$. It follows from the growth condition (4.2) that $\widetilde{f}(u)=f(\cdot, u(\cdot)) \in L_{\Phi_{0}^{*}}$ for every $u \in L_{\Phi_{0}}$ and the mapping $\widetilde{f}: u \mapsto f(\cdot, u(\cdot))$ is continuous and bounded from $L_{\Phi_{0}}$ into $L_{\Phi_{0}^{*}}$. Let

$$
\mathcal{F}=i^{*} \tilde{f} i \text {. }
$$

In this case (4.1) is equivalent to a fixed point inclusion in $L_{\Phi_{0}}$.

Proposition 4.1. Let $\mathcal{F}$ be given by (4.3) with $f$ satisfying (4.2). If $u$ is a solution of (4.1) then $w=i u$ is a solution of the fixed point inclusion

$$
w \in L_{\Phi_{0}}: w \in\left(i S_{K} i^{*} \widetilde{f}\right)(w) .
$$

Conversely, if $w$ is a solution of (4.4) then $w \in X$ and is a solution of (4.1).

ProOF. If $u$ is a solution of (4.1) then $w=i w \in L_{\Phi_{0}}$ satisfies $w=i u \in$ $i S_{K}[\mathcal{F}(u)]=i S_{K}\left[i^{*} \widetilde{f}(i u)\right]=\left(i S_{K} i^{*} \widetilde{f}\right)(w)$. Conversely, assume $w$ is a solution of (4.4). Since $S_{K}\left(X^{*}\right) \subset K \subset X$, we have $w \in K \subset X$. Hence, $w=i w \in$ $i S_{K}\left[i^{*} \widetilde{f} i(w)\right]=S_{K}[\mathcal{F}(w)]$, i.e. $w$ satisfies (4.1).

Some useful properties of $i S_{K} i^{*} \tilde{f}$ and thus of the formulation (4.4) are given in the following result.

Proposition 4.2. (a) For each $w \in L_{\Phi_{0}},\left(i S_{K} i^{*} \widetilde{f}\right)(w)$ is a nonempty, convex, compact subset of $L_{\Phi_{0}}$.

(b) The mapping $i S_{K} i^{*} \widetilde{f}$ is upper semicontinuous and compact from $L_{\Phi_{0}}$ into $2^{L_{\Phi_{0}}} \backslash\{\emptyset\}$.

Proof. (a) For any $L \in X^{*}$, since $S_{K}(L)$ is nonempty, convex, closed, and bounded in $X$, and $i$ is compact, it is straightforward to see that $\left(i S_{K} i^{*} \widetilde{f}\right)(w)$ is nonempty, convex, and compact in $L_{\Phi_{0}}$.

(b) Let $w_{0} \in L_{\Phi_{0}}$ and $U$ be an open set in $L_{\Phi_{0}}$ such that

$$
\left(i S_{K} i^{*} \widetilde{f}\right)\left(w_{0}\right) \subset U \text {. }
$$

Since the embedding $i$ is compact, $i^{-1}(U)=U \cap X$ is weakly open in $X$. Furthermore, $\left(S_{K} i^{*} \widetilde{f}\right)\left(w_{0}\right) \subset i^{-1}(U)=U \cap X$. From Theorem 3.7(c), there exists an open neighbourhood $W$ of $i^{*} \widetilde{f}\left(w_{0}\right)$ in $X^{*}$ such that $S_{K}(W)=\bigcup_{L \in W} S_{K}(L) \subset$ $U \cap X$. Since $\tilde{f}$ is continuous from $L_{\Phi_{0}}$ to $L_{\Phi_{0}^{*}}$ and $i^{*}$ is continuous from $L_{\Phi_{0}^{*}}$ to $X^{*}, V=\left(i^{*} \widetilde{f}\right)^{-1}(W)$ is an open neighbourhood of $w_{0}$ in $L_{\Phi_{0}}$. If $w \in V$ then $i^{*} \widetilde{f}(w) \in W$ and thus $S_{K}\left(i^{*} \widetilde{f}(w)\right) \subset U \cap X$. This means that $\left(i S_{K} i^{*} \widetilde{f}\right)(V) \subset U$, proving the upper semicontinuity of $i S_{K} i^{*} \tilde{f}$. 
Assume now that $W$ is a bounded set in $L_{\Phi_{0}}$. Since $\widetilde{f}$ is a bounded mapping, $\widetilde{f}(W)$ is bounded in $L_{\Phi_{0}^{*}}$ and thus $i^{*} \widetilde{f}(W)$ is bounded in $X^{*}$. Because of the boundedness of $S_{K}$, we see that $S_{K} i^{*} \widetilde{f}(W)$ is bounded in $X$. Since $i$ is compact, $\left(i S_{K} i^{*} \widetilde{f}\right)(W)$ is relatively compact in $L_{\Phi_{0}}$. This proves that $i S_{K} i^{*} \widetilde{f}$ is a (multivalued) compact mapping.

The properties of $i S_{K} i^{*} \widetilde{f}$ in Proposition 4.2 allow us to use classical topological tools such the Tikhonov's fixed point theorem for multivalued compact vector fields or variants of topological degrees of multivalued perturbations of identity to study the existence and other behaviors of solutions of (4.4), cf. e.g. [2], [43], [15]. For example, Proposition 4.2 implies that the topological degree (cf. e.g. [39], [42]) $\operatorname{deg}\left(I-i S_{K} i^{*} \widetilde{f}, U, a\right)$ is defined, where $I$ is the identity mapping of $L_{\Phi_{0}}, U$ is an open bounded subset of $L_{\Phi_{0}}$ and $a \in L_{\Phi_{0}} \backslash\left(I-i S_{K} i^{*} \widetilde{f}\right)(\partial U)$. We have therefore the following basic existence and localization theorem for the inclusion (4.4) in $L_{\Phi_{0}}$ and thus for the variational inequality (3.19).

Theorem 4.3. Assume $U$ is an open bounded subset of $L_{\Phi_{0}}$ such that

$$
0 \notin u-\left(i S_{K} i^{*} \tilde{f}\right)(u), \quad \text { for all } u \in \partial U,
$$

and $\operatorname{deg}\left(I-i S_{K} i^{*} \widetilde{f}, U, 0\right) \neq 0$. Then the inclusion (4.4) and thus the variational inequality (3.19) have solutions in $K \cap U$.

\section{An example}

We conclude this paper with a more concrete example and prove an existence theorem when $f$ is the sum of a "subcritical" signed function and a "sublinear" one.

Let $F$ be a Young function from $\mathbb{R}$ to $[0, \infty]$. We denote by $\widehat{F}$ the function from $\mathbb{R}^{N}$ to $[0, \infty]$ defined by

$$
\widehat{F}(\xi)=F(|\xi|), \quad \text { for all } \xi \in \mathbb{R}^{N} .
$$

It is easy to see that $\widehat{F}$ is a $G$-function (i.e. $\widehat{F}$ satisfies (G1)-(G6)) and if $F$ is an $N$-function then $\widehat{F}$ satisfies also (G2') and (G5').

Assume

$$
f=g+h,
$$

where $g$ and $h$ are Carathéodory functions from $\Omega \times \mathbb{R}$ into $\mathbb{R}$. The sublinearity (with respect to $\Phi$ ) of $g$ is extended from the classical case Sobolev spaces to our case as follows:

(G) There are $N$-functions $\Psi$ and $\Psi_{0}$ such that

$$
\begin{aligned}
\Psi_{0} & \ll \Psi, \\
\widehat{\Psi} & \prec \Phi,
\end{aligned}
$$


and

$$
|g(x, u)| \leq B_{1}(x)+C_{1}\left[\left(\Psi_{0}^{*}\right)^{-1} \Psi_{0}\right](|u|),
$$

for almost every $x \in \Omega$, all $u \in \mathbb{R}$, where $C_{1}>0$ and $B_{1} \in L_{\Psi_{0}^{*}}$.

Note that condition $(\mathrm{G})$ is an extension of the usual sublinear growth condition to our case. Concerning $h$, we assume that:

(H) $h$ has subcritical growth (as in (4.2)):

$$
|h(x, u)| \leq B_{2}(x)+C_{2}\left[\left(\Phi_{0}^{*}\right)^{-1} \Phi_{0}\right](|u|),
$$

for almost every $x \in \Omega$, all $u \in \mathbb{R}$, where $C_{2}>0$ and $B_{2} \in L_{\Phi_{0}^{*}}$, and

$$
h(x, u) u \geq 0, \quad \text { for a.e. } x \in \Omega \text {, all } u \in \mathbb{R} .
$$

From (5.3), we see that $X$ is continuously embedded into the Orlicz-Sobolev space $W_{0}^{1} L_{\widehat{\Psi}}=W_{0}^{1} L_{\Psi}$. From (5.2), $W_{0}^{1} L_{\Psi}$ is compactly embedded into $L_{\Psi_{0}}$. Hence, the embedding $i_{\Psi_{0}}: X \hookrightarrow L_{\Psi_{0}}$ is compact. On the other hand, as discussed above, the embedding $i_{\Phi_{0}}: X \hookrightarrow L_{\Phi_{0}}$ is also compact. Furthermore, the growth conditions (5.4) and (5.5) imply that the Niemytskii operators $\widetilde{g}: u \mapsto g(\cdot, u(\cdot))$ and $\widetilde{h}: u \mapsto h(\cdot, u(\cdot))$ are bounded and thus continuous from $L_{\Psi_{0}}$ into $L_{\Psi_{0}^{*}}$ and from $L_{\Phi_{0}}$ into $L_{\Phi_{0}^{*}}$, respectively. The mapping $\mathcal{F}$ given by (3.15) with $f$ given by (5.1) is well defined and moreover, $\mathcal{F}$ can be written in this case as

$$
\mathcal{F}=i_{\Psi_{0}}^{*} \widetilde{g} i_{\Psi_{0}}+i_{\Phi_{0}}^{*} \widetilde{h} i_{\Phi_{0}} .
$$

We have the following existence result for (3.19).

THEOREM 5.1. Assume $\mathcal{F}$ is given by (3.15) and (5.1) with $g$ and $h$ satisfying $(\mathrm{G})$ and $(\mathrm{H})$. Then the variational inequality (3.19) has solutions.

Proof. We shall apply Theorem 3.4 by checking the assumptions there. First, note that since $i_{\Psi_{0}}, i_{\Phi_{0}}$ are compact and $\widetilde{g}, \widetilde{h}$ are continuous on their domains, the mappings $i_{\Psi_{0}}^{*} \widetilde{g} i_{\Psi_{0}}, i_{\Phi_{0}}^{*} \widetilde{h} i_{\Phi_{0}}$, and thus $\mathcal{F}$ are completely continuous from $X$ into $X^{*}$ (i.e. continuous from $X$ with the weak topology to $X^{*}$ with the strong topology). This immediately implies that $\mathcal{F}$ is pseudomonotone. We only need to verify the coercivity condition (3.22) with $u_{0}=0$ and $R$ sufficiently large. In fact, we have for any $u \in X$,

$$
\begin{aligned}
\langle\mathcal{F}(u), u\rangle+J(u) & =\int_{\Omega} \Phi(\nabla u) d x+\int_{\Omega} g(x, u) u d x+\int_{\Omega} h(x, u) u d x \\
& \geq \int_{\Omega} \Phi(\nabla u) d x+\int_{\Omega} g(x, u) u d x .
\end{aligned}
$$

From (5.3), there exist $k_{1}, \alpha_{0}, \beta_{0}>0$ such that $\Phi\left(k_{1} \xi\right) \geq \alpha_{0} \widehat{\Psi}(\xi)-\beta_{0}=$ $\alpha_{0} \Psi(|\xi|)-\beta_{0}$ for all $\xi \in \mathbb{R}^{N}$. Since $\Phi$ satisfies a $\Delta_{2}$ condition at $\infty$, this implies 
the existence of $\alpha_{1}, \beta_{1}>0$ such that

$$
\Phi(\xi) \geq \alpha_{1} \Psi(|\xi|)-\beta_{1}, \quad \text { for all } \xi \in \mathbb{R}^{N} .
$$

It follows from Poincaré's inequality for Orlicz-Sobolev spaces (Lemma 5.7, [16]) that

$$
\int_{\Omega} \Psi(|\nabla v|) d x \geq \alpha_{3} \int_{\Omega} \Psi\left(k_{2}|v|\right) d x, \quad \text { for all } v \in W_{0}^{1} L_{\Psi},
$$

for some constants $k_{2}, \alpha_{3}>0$. Since $u \in X \hookrightarrow W_{0}^{1} L_{\Psi},(5.9)$ and (5.10) give

$$
\int_{\Omega} \Phi(\nabla u) d x \geq \alpha_{1} \int_{\Omega} \Psi(|\nabla u|) d x-\beta_{1}|\Omega| \geq \alpha_{4} \int_{\Omega} \Psi\left(k_{2}|u|\right) d x-\beta_{4} .
$$

On the other hand, (5.4) and Young's inequality imply

$$
\begin{aligned}
& \left|\int_{\Omega} g(x, u) u d x\right| \leq \int_{\Omega} B_{1}|u| d x+C_{1} \int_{\Omega}\left(\Psi_{0}^{*}\right)^{-1} \Psi_{0}(|u|)|u| d x \\
& \quad \leq C_{3}\|u\|_{L_{\Psi_{0}}}+C_{1} \int_{\Omega}\left\{\Psi_{0}^{*}\left[\left(\Psi_{0}^{*}\right)^{-1} \Psi_{0}(|u|)\right]+\Psi_{0}(|u|)\right\} d x \\
& \quad \leq C_{4}\|u\|+2 C_{1} \int_{\Omega} \Psi_{0}(|u|) d x .
\end{aligned}
$$

Given any $\varepsilon>0$, from (5.2) there exists $\beta_{5}>0$ such that

$$
\Psi_{0}(|s|) \leq \varepsilon \Psi\left(k_{2}|s|\right)+\beta_{5}, \quad \text { for all } s \in \mathbb{R} .
$$

Combining (5.12), (5.13) with (5.11) yields

$$
\begin{aligned}
&\left|\int_{\Omega} g(x, u) u d x\right| \leq C_{4}\|u\|+2 \varepsilon C_{1} \int_{\Omega} \Psi\left(k_{2}|u|\right) d x+2 C_{1} \beta_{5}|\Omega| \\
& \leq C_{4}\|u\|+\frac{2 \varepsilon C_{1}}{\alpha_{4}} \int_{\Omega} \Phi(\nabla u) d x+\left(2 C_{1} \beta_{5}|\Omega|+\frac{2 \varepsilon C_{1} \beta_{4}}{\alpha_{4}}\right) .
\end{aligned}
$$

Choosing $\varepsilon=\alpha_{4} /\left(4 C_{1}\right)$, we obtain from (5.8) and (5.14) that

$$
\langle\mathcal{F}(u), u\rangle+J(u) \geq \frac{1}{2} \int_{\Omega} \Phi(\nabla u) d x-C_{4}\|u\|-\left(2 C_{1} \beta_{5}|\Omega|+\frac{\beta_{4}}{2}\right) .
$$

As a consequence of Proposition 2.7, the right hand side of this inequality is positive for all $u \in X$ with $\|u\|$ sufficiently large. Therefore, both conditions (3.21) and (3.22) hold for $R$ sufficiently large. According to Theorem 3.4, (3.19) has solutions.

REMARK 5.2. (a) We can get a similar result to Theorem 5.1 by using alternatively topological degrees and Theorem 4.3. As noted in Remark 3.6, by following this approach we only need to assume that $G^{*}$ satisfies a $\Delta_{2}$ condition and thus can extend several results in [36] to variational inequalities in anisotropic Orlicz-Sobolev spaces.

(b) We present here a monotonicity and a topological/fixed point method to study the existence of solutions of (1.1); other approaches such as variational, 
topological, and sub-supersolution ones on problems with other boundary conditions will be the subjects of some forthcoming projects. Regarding to the relaxation of the $\Delta_{2}$ conditions, we hope that the arguments used in problems without such conditions in (isotropic) non-reflexive Orlicz-Sobolev spaces that we studied before in e.g. [14], [27]-[32], [34]-[38] could be extended to problems in anisotropic Orlicz-Sobolev spaces or Orlicz-Musielak-Sobolev spaces. Such extensions are not within the scope of this introductory paper and would be investigated in some future works.

Acknowledgments. The author would like to thank the referee for his valuable remarks and additional references.

\section{REFERENCES}

[1] R. Adams, Sobolev spaces, Academic Press, New York, 1975.

[2] R.P. Agarwal, M. Meehan and D. O'Regan, Fixed point theory and applications, Cambridge Tracts in Mathematics, vol. 141, Cambridge University Press, Cambridge, 2001.

[3] J.-P. Aubin, Optima and equilibria, Graduate Texts in Mathematics, vol. 140, SpringerVerlag, Berlin, 1993.

[4] M.-M. Boureanu, P. PuCCI ANd V. RĂdulescu, Multiplicity of solutions for a class of anisotropic elliptic equations with variable exponent, Complex Var. Elliptic Equ. 56 (2011), 755-767.

[5] K. Chelminski and P. Gwiazda, Convergence of coercive approximations for strictly monotone quasistatic models in inelastic deformation theory, Math. Methods Appl. Sci. 30 (2007), no. 12, 1357-1374.

[6] A. Cianchi, A sharp embedding theorem for Orlicz-Sobolev spaces, Indiana Univ. Math. J. 45 (1996), no. 1, 39-65.

[7] _ A fully anisotropic Sobolev inequality, Pacific J. Math. 196 (2000), no. 2, 283-295.

[8] _ Optimal Orlicz-Sobolev embeddings, Rev. Mat. Iberoamericana 20 (2004), no. 2, $427-474$

[9] W. Desch AND R. GRImmer, On the wellposedness of constitutive laws involving dissipation potentials, Trans. Amer. Math. Soc. 353 (2001), no. 12, 5095-5120.

[10] T. Donaldson and N. Trudinger, Orlicz-Sobolev spaces and imbedding theorems, J. Functional Analysis 8 (1971), 52-75.

[11] I. Ekeland and R. Temam, Analyse convexe et problèmes variationnels, Dunod, 1974.

[12] XIAnling FAn, Anisotropic variable exponent Sobolev spaces and $p(x)$-Laplacian equations, Complex Var. Elliptic Equ. 56 (2011), 623-642.

[13] Xianling Fan and Chun-Xia Guan, Uniform convexity of Musielak-Orlicz-Sobolev spaces and applications, Nonlinear Anal. 73 (2010), no. 1, 163-175.

[14] M. García-Huidobro, V.K. Le, R. ManÁsevich and K. Schmitt, On pricipal eigenvalues for quasilinear elliptic differential operators: An Orlicz-Sobolev space setting, Nonlinear Diff. Eq. Appl. 6 (1999), 207-225.

[15] L. GóRniewicz, Topological fixed point theory of multivalued mappings, Topological Fixed Point Theory and Its Applications, vol. 4, Springer, Dordrecht, 2006.

[16] J.P. Gossez, Nonlinear elliptic boundary value problems for equations with rapidly or slowly increasing coefficients, Trans. Amer. Math. Soc. 190 (1974), 163-205.

[17] P. Gwiazda, P. Minakowski and A. Wróblewska-Kaminska, Elliptic problems in generalized Orlicz-Musielak spaces, Cent. Eur. J. Math. 10 (2012), 2019-2032. 
[18] P. Gwiazda And A. Świerczewska-Gwiazda, On non-Newtonian fluids with a property of rapid thickening under different stimulus, Math. Models Methods Appl. Sci. 18 (2008), 1073-1092.

[19] _ On steady non-Newtonian fluids with growth conditions in generalized Orlicz spaces, Topol. Methods Nonlinear Anal. 32 (2008), no. 1, 103-113.

[20] P. Gwiazda, A. Świerczewska-Gwiazda and A. WróBlewska, Monotonicity methods in generalized Orlicz spaces for a class of non-Newtonian fluids, Math. Methods Appl. Sci. 33 (2010), 125-137.

[21] _ Generalized Stokes system in Orlicz spaces, Discrete Contin. Dyn. Syst. 32 (2012), no. $6,2125-2146$.

[22] P. Gwiazda, P. Wittbold, A. Wróblewska and A. Zimmermann, Renormalized solutions of nonlinear elliptic problems in generalized Orlicz spaces, J. Differential Equations $253(2012)$.

[23] E. Hewitt and K. Stromberg, Real and abstract analysis. A modern treatment of the theory of functions of a real variable, Springer, 1965.

[24] A. KozeK, Orlicz spaces of functions with values in Banach spaces, Comment. Math. Prace Mat. 19 (1976/77), no. 2, 259-288.

[25] _ Convex integral functionals on Orlicz spaces, Comment. Math. Prace Mat. 21 (1980), no. 1, 109-135.

[26] M.A. Krasnosels'KII and J. Rutic'KIĬ, Convex functions and Orlicz spaces, Noordhoff, Groningen, 1961

[27] V. K. LE, Some existence results for noncoercive nonconvex minimization problems with fast or slow perturbing terms, Num. Func. Anal. and Optim. 20 (1999), 37-58.

[28] _ Nontrivial solutions of mountain pass type of quasilinear equations with slowly growing principal parts, J. Diff. Int. Eq. 15 (2002), 839-862.

[29]___ Generic existence result for an eigenvalue problem with rapidly growing principal operator, ESAIM Control Optim. Calc. Var. 10 (2004), no. 4, 677-691.

[30] _ Some existence and bifurcation results for quasilinear elliptic equations with slowly growing principal operators, Houston J. Math. 32 (2006), no. 3, 921-943.

[31] _ Sub-supersolutions in a variational inequality related to a sandpile problem, ANZIAM J. 48 (2006), no. 2, 179-197.

[32] _ Some existence results and properties of solutions in quasilinear variational inequalities with general growths, Differ. Equ. Dyn. Syst. 17 (2009), 343-364.

[33] _ A range and existence theorem for pseudomonotone perturbations of maximal monotone operators, Proc. Amer. Math. Soc. 139 (2011), 1645-1658.

[34] V. K. Le ANd D. Motreanu, On nontrivial solutions of variational-hemivariational inequalities with slowly growing principal parts, Z. Anal. Anwend. 28 (2009), 277-293.

[35] V.K. Le, D. Motreanu And V.V. Motreanu, On a non-smooth eigenvalue problem in Orlicz-Sobolev spaces, Appl. Anal. 89 (2010), 229-242.

[36] V.K. Le AND K. Schmitt, Quasilinear elliptic equations and inequalities with rapidly growing coefficients, J. London Math. Soc. 62 (2000), 852-872.

[37] Some general concepts of sub- and supersolutions for nonlinear elliptic problems, Topol. Methods Nonlinear Anal. 28 (2006), 87-103.

[38] __ Equations and inequalities in Orlicz-Sobolev spaces: Selected topics, pp. 295-351, International Press, Boston, 2010.

[39] N.G. LlOYd, Degree theory, Cambridge University Press, 1978.

[40] W.A.J. Luxemburg, Banach function spaces, Thesis, Technische Hogeschool te Delft, 1955. 
[41] M. MihăIlescu, G. Moroşanu And V. RĂDulescu, Eigenvalue problems for anisotropic elliptic equations: an Orlicz-Sobolev space setting, Nonlinear Anal. 73 (2010), 3239-3253.

[42] D. O'Regan, Y.J. Cho And Y.Q. Chen, Topological degree theory and applications, Series in Mathematical Analysis and Applications, vol. 10, Chapman \& Hall/CRC, Boca Raton, FL, 2006.

[43] D. O'Regan and R. Precup, Theorems of Leray-Schauder type and applications, Series in Mathematical Analysis and Applications, vol. 3, Gordon and Breach Science Publishers, Amsterdam, 2001.

[44] W. Pompe, Existence theorems in the viscoplasticity theory, Doctoral thesis, Aachen, Shaker Verlag, 2003.

[45] T.R. Rockafellar and R.J. Wets, Variational analysis, Springer, Berlin, 1998.

[46] G. Schappacher, A notion of Orlicz spaces for vector valued functions, Appl. Math. 50 (2005), no. 4, 355-386.

[47] N.S. Trudinger, An imbedding theorem for $H^{0}(G, \Omega)$ spaces, Studia Math. 50 (1974), $17-30$.

Vy KHoi Le

Department of Mathematics and Statistics

Missouri University of Science and Technology

Rolla, MO 65409, USA

E-mail address: vy@mst.edu 